TRANSACTIONS OF THE

AMERICAN MATHEMATICAL SOCIETY

Volume 359, Number 6, June 2007, Pages 2687-2719

S 0002-9947(07)03997-9

Article electronically published on January 25, 2007

\title{
CLASSIFYING REPRESENTATIONS BY WAY OF GRASSMANNIANS
}

\author{
BIRGE HUISGEN-ZIMMERMANN
}

Dedicated to the memory of Sheila Brenner

\begin{abstract}
Let $\Lambda$ be a finite-dimensional algebra over an algebraically closed field. Criteria are given which characterize existence of a fine or coarse moduli space classifying, up to isomorphism, the representations of $\Lambda$ with fixed dimension $d$ and fixed squarefree top $T$. Next to providing a complete theoretical picture, some of these equivalent conditions are readily checkable from quiver and relations of $\Lambda$. In the case of existence of a moduli space- unexpectedly frequent in light of the stringency of fine classification - this space is always projective and, in fact, arises as a closed subvariety $\mathfrak{G r a s s}_{d}^{T}$ of a classical Grassmannian. Even when the full moduli problem fails to be solvable, the variety $\mathfrak{G r a s s}_{d}^{T}$ is seen to have distinctive properties recommending it as a substitute for a moduli space. As an application, a characterization of the algebras having only finitely many representations with fixed simple top is obtained; in this case of 'finite local representation type at a given simple $T$ ', the radical layering $\left(J^{l} M / J^{l+1} M\right)_{l \geq 0}$ is shown to be a classifying invariant for the modules with top $T$. This relies on the following general fact obtained as a byproduct: proper degenerations of a local module $M$ never have the same radical layering as $M$.
\end{abstract}

\section{IntRoduction AND TERMINOLOGY}

Throughout, $\Lambda$ denotes a finite-dimensional algebra over an algebraically closed field $K$. While the full representation-theoretic picture of $\Lambda$ is beyond the scope of a complete description if $\Lambda$ has wild representation type, substantial portions of this representation theory do lie within reach in many wild situations. The part we address here consists of the representations $M$ of fixed dimension $d$ that have fixed squarefree top $T=M / J M$, where $J$ is the Jacobson radical of $\Lambda$; in other words, we require that the simple (left) $\Lambda$-modules occur with multiplicity at most 1 in $T$. Our primary goal is to decide when the restricted classification problem for isomorphism classes of $d$-dimensional representations with top $T$ has a coarse or fine moduli space. In rough terms, this means that we seek to bijectively parametrize these isomorphism classes by the points of an algebraic variety such that the structure constants 'evolve Zariski continuously' as one moves along the parameter space - this continuity condition is made precise in the concept of a family - and so that other continuous parametrizations are uniquely induced by the distinguished one - the latter requirement is made precise via universal properties of

Received by the editors April 20, 2004 and, in revised form, March 21, 2005.

2000 Mathematics Subject Classification. Primary 16G10, 16G20, 16G60, 14D20, 14D22.

This research was partially supported by a grant from the National Science Foundation.

(C)2007 American Mathematical Society Reverts to public domain 28 years from publication 
varying degrees of stringency. (This classification philosophy actually predates the rigorous definition of coarse and fine moduli spaces given by Mumford in the 1960s; it already underlies Riemann's classification of nonsingular projective curves in the 1850s, where the term 'moduli' was coined, standing for a 'structure-determining collection of continuous parameters in $\mathbb{C}^{\prime}$.) A precise definition of a fine moduli space can be found at the end of Section 1.

In our situation, the existence problems for fine and coarse moduli spaces will turn out to be equivalent. One of our main objectives is to provide readily verifiable necessary and sufficient conditions for existence. In the positive case, we exhibit the fine moduli space - it is always projective - together with the universal family that classifies, up to isomorphism, the $d$-dimensional modules with top $T$.

In the classical affine variety $\operatorname{Mod}_{d}^{\Lambda}$ whose $\mathrm{GL}_{d}$-orbits bijectively parametrize the isomorphism classes of $d$-dimensional $\Lambda$-modules, all orbits corresponding to nonsemisimple modules fail to be closed. Therefore, the standard methods of invariant theory, typically restricting attention to the closed orbits, are a priori not helpful. In a seminal article, [12, King coped with this difficulty by adapting Mumford's concept of stability of vector bundles on projective curves, which led him to focus on $\Lambda$-modules which are '(semi)stable' relative to a given additive function $\Theta: K_{0}(\Lambda$ - mod $) \rightarrow \mathbb{R}$. For the $\Theta$-stable modules, a fine moduli space classifying up to isomorphism is guaranteed, but this class of representations is often hard to identify or assess in size.

Here we initiate a quite different approach to the moduli problem. Let us start by presenting a projective variety whose points always parametrize, not necessarily bijectively, the $d$-dimensional representations with fixed top $T$. As in the classical setting, the isomorphism classes are again in 1-1 correspondence with the orbits of an algebraic group action, but these orbits have lower dimension and are closed much more frequently. This variety was first introduced by Bongartz and the author in [5] and [6]. Fix $d$ and a semisimple module $T$, not yet assumed to be squarefree, with projective cover $P$. We denote by $\mathfrak{G r a s s}_{d}^{T}$ the closed subvariety of the classical Grassmannian of all $(\operatorname{dim} P-d)$-dimensional subspaces of $J P$ that consists of the $\Lambda$-submodules of $J P$ of dimension $\operatorname{dim} P-d$. Clearly, $\mathfrak{G r a s s}_{d}^{T}$ is a projective variety endowed with a morphic action of the automorphism group $\operatorname{Aut}_{\Lambda}(P)$, the orbits of which coincide with the fibres of the surjection

$$
\phi: \mathfrak{G r a s s}_{d}^{T} \longrightarrow\{\text { isomorphism types of } d \text {-dimensional modules with top } T\}
$$

sending $C$ to the isomorphism type of $P / C$. See Section 2 for basic facts about the variety $\mathfrak{G}_{\mathfrak{r a s s}} \mathfrak{a n d}_{d}^{T}$ and its relationship to its classical counterpart.

From now on, we assume $T$ to be squarefree. Without this hypothesis, the solution to the moduli problem requires modification and an additional layer of machinery. It will be treated in a sequel to the present work; a brief summary is given in Section 7. Adopting the notion of a 'family of $\Lambda$-modules' introduced by King (cf. [12] and the last paragraph of Section 1), we obtain the following characterization of the triples $\Lambda, T, d$ for which the moduli problem has a solution (see Theorems 4.2 and 4.4 for somewhat stronger results and Section 2.A for the concept of degeneration):

Theorem A. The following statements are equivalent for squarefree $T$.

(1) There exists a coarse moduli space classifying the d-dimensional $\Lambda$-modules with top T, up to isomorphism. 
(2) There exists a fine moduli space classifying the $d$-dimensional $\Lambda$-modules with top $T$, up to isomorphism.

(3) No d-dimensional $\Lambda$-module with top $T$ has a proper degeneration with top $T$.

(4) Every submodule $C \subseteq J P$ of codimension $d$ in $P$ is invariant under all endomorphisms of $P$.

(5) $\mathfrak{G r a s s}_{d}^{T}$ is the fine moduli space for the d-dimensional $\Lambda$-modules with top $T$ (in particular, all $\operatorname{Aut}_{\Lambda}(P)$-orbits of $\mathfrak{G r a s s}_{d}^{T}$ are singletons).

We emphasize that Theorem A addresses the existence of a moduli space for the isomorphism classes of all $d$-dimensional $\Lambda$-modules with top $T$; in particular, no stability conditions are imposed.

The equivalence of (3) and (4) holds 'pointwise', in the following sense: an individual module $M$ with top $T$, say $M=P / C$, is devoid of proper top- $T$ degenerations precisely when $C$ is fully invariant in $P$; the former condition is, a priori, hard to check, whereas recognizing full invariance is easy. Invariance is a strong requirement, but nonetheless globally satisfied for the submodules of $J P$ of fixed codimension in a wide range of interesting cases; see Corollary 4.5 for illustration. (For the sake of contrast, if $d \geq 2$, the full collection of $d$-dimensional modules has a coarse moduli space only when $\Lambda$ is semisimple.)

When $T$ is simple and the Gabriel quiver of $\Lambda$ has no oriented cycles, the existence of fine moduli spaces for representations with fixed top $T$ can alternatively be deduced from King's method; for details, see the comments following Theorem 4.4. Moreover, we refer to work of Le Bruyn and Schofield, [14] and 20], for results concerning the structure of moduli spaces over hereditary algebras in the case of existence; in particular, Schofield provides very general sufficient conditions for rationality in that case 20 .

One of the representation-theoretically most useful features of $\mathfrak{G r a s s}_{d}^{T}$ - here and in subsequent work - is a finite affine cover, $(\mathfrak{G} \mathfrak{r a s s}(\sigma))_{\sigma}$, with the following properties (see Theorem 3.5): (a) It consists of $\operatorname{Aut}_{\Lambda}(P)$-stable charts, and (b) it is the disjoint union of open affine covers of those subvarieties $\mathfrak{G r a s s}(\mathbb{S})$ of $\mathfrak{G} \mathfrak{r a s s} \mathfrak{s}_{d}^{T}$ which consist of the points representing modules $M$ with fixed radical layering $\mathbb{S}=\left(J^{l} M / J^{l+1} M\right)_{l>0}$; the latter is meaningful as we identify isomorphic semisimple modules. For a bit more detail, suppose $\Lambda=K Q / I$, where $Q$ is a quiver and $I$ an admissible ideal in the path algebra $K Q$. In this scenario, each chart $\mathfrak{G} \mathfrak{r a s s}(\sigma)$ is indexed by a set $\sigma$ of paths tied to the algebraic structure of the $\Lambda$-modules corresponding to the points of $\mathfrak{G} \mathfrak{r a s s}(\sigma)$. The cover $(\mathfrak{G} \mathfrak{r a s s}(\sigma))_{\sigma}$ is distinguished by its functoriality relative to the ideal $I$ of relations of $\Lambda$, the pertinent functor depending only on $Q, d$, and the set of vertices determining $T$ (see Section 3.17). We add a few comments relating the $\mathfrak{G} \mathfrak{r a s s}(\sigma)$ to the Schubert cells of the encompassing full Grassmannian $\operatorname{Gr}(\operatorname{dim} P-d, J P)$. The intersections of $\mathfrak{G r a s s}_{d}^{T}$ with Schubert cells usually fail to satisfy the above conditions (a) and (b). In particular, the intersections with open Schubert cells are hardly ever stable under the $\operatorname{Aut}_{\Lambda}(P)$-action. On the other hand, each chart $\mathfrak{G} \mathfrak{r a s s}(\sigma)$ embeds into a suitable open Schubert cell of $\operatorname{Gr}(\operatorname{dim} P-d, J P)$, and the affine coordinates of $\mathfrak{G r a s s}(\sigma)$ introduced in Section 3.10 are essentially the Plücker coordinates that correspond to this embedding. However, the Plücker coordinates can be pared down to a comparatively small subfamily encoding all relevant information, due to our specific algebraic setting; it is this economy that permits comparatively effortless analysis of even large examples 
(see [10]). While the cover $(\mathfrak{G r a s s}(\sigma))_{\sigma}$ provides the backbone for our proofs, familiarity with its specifics is not required for an understanding of the main results. The reader only interested in the theorems and their theoretical applications is therefore encouraged to skip the somewhat technical portion of 3.B following Theorem 3.5.

Polynomials defining any of the affine subvarieties $\mathfrak{G} \mathfrak{r a s s}(\sigma)$ of $\mathfrak{G r a s s}_{d}^{T}$ are available from quiver and relations of $\Lambda$ by way of easy combinatorial manipulations (Section 3.13). These polynomials provide the foundation for all of our concrete examples here and in [10; in particular, they make all of the geometric conditions arising in the theorems checkable through Gröbner methods (see 1]). Furthermore, they establish a combinatorial link between the geometry of the affine charts $\mathfrak{G r a s s}(\sigma)$ and algebraic features of the classes of representations parametrized by them (see, e.g., [10, Section 5).

In situations where the $d$-dimensional $\Lambda$-modules with fixed top fail to have a moduli space, the natural next step is to subdivide the target class by fixing further discrete invariants. There is an obvious refinement of our primary partition of the $d$-dimensional representations $M$ in terms of tops: namely, the partition in terms of full radical layerings $\mathbb{S}=\mathbb{S}(M)=\left(J^{l} M / J^{l+1} M\right)_{0 \leq l \leq L}$, where $L$ is chosen so that $J^{L+1}=0$; in other words, we fix the matrix recording the multiplicities of all simple composition factors of $M$ in a format that keeps track of their 'layer-locations'. The main benefit of restricting one's focus to $\mathfrak{G} \mathfrak{r a s s}(\mathbb{S})$ lies in the fact that the relative closures of the $\operatorname{Aut}_{\Lambda}(P)$-orbits in $\mathfrak{G r a s s}(\mathbb{S})$ are better understood than the closures in $\mathfrak{G r a s s}_{d}^{T}$ (see Section 4); in particular, the orbits of $\mathfrak{G} \mathfrak{r a s s}(\mathbb{S})$ under the action of the unipotent radical of $\operatorname{Aut}_{\Lambda}(P)$ are always relatively closed. When specialized to the case of a simple top $T$, this yields:

Theorem B (see Section 2.A for terminology). No $\Lambda$-module $M$ with simple top has a proper degeneration $M^{\prime}$ with $J^{l} M / J^{l+1} M \cong J^{l} M^{\prime} / J^{l+1} M^{\prime}$ for $0 \leq l \leq L$.

Section 4 also displays a first set of reasons for viewing $\mathfrak{G r a s s}_{d}^{T}$, and a fortiori $\mathfrak{G} \mathfrak{r a s s}(\mathbb{S})$, as a useful approximation to a moduli space for the corresponding classification problem whenever the moduli problem in Mumford's sense is not solvable (for further backing of this viewpoint, we refer to [10]). To name one reason, the fibres $\operatorname{Aut}_{\Lambda}(P) . C$ of the map $\phi$ are well-understood in terms of their intrinsic structure: if $T$ is simple, the $\operatorname{Aut}_{\Lambda}(P)$-orbits are affine spaces $\mathbb{A}^{m}$, where $m$ is bounded above by the multiplicity of $T$ in $J P$. For general squarefree $T$, each orbit $\operatorname{Aut}_{\Lambda}(P) . C$ is a direct product of an affine space $\mathbb{A}^{m}$ and a torus $\left(K^{*}\right)^{r}$, where $\operatorname{dim} T-r$ is the number of indecomposable summands of $P / C$.

Our characterization of the algebras that have finite local representation type with respect to a simple module $T$, as defined in the abstract, hinges on Theorem B. It lends additional support to the central role we attribute to the radical layering as a discrete invariant of a representation. Condition (3) below is decidable from quiver and relations of $\Lambda$ by way of the mentioned polynomials; if it is satisfied, the modules with top $T$ can be explicitly constructed from these data. See Theorem 5.2 for additional information.

Theorem C. For any simple $\Lambda$-module $T$, the following conditions are equivalent (they are not left-right symmetric - we refer to left modules, say):

(1) There are only finitely many $\Lambda$-modules with top $T$, up to isomorphism.

(2) If $M$ and $N$ are $\Lambda$-modules with top $T$, then $M \cong N$ if and only if $\mathbb{S}(M)=$ $\mathbb{S}(N)$. 
(3) For every $d \in \mathbb{N}$ and every sequence $\mathbb{S}=\left(\mathbb{S}_{0}, \mathbb{S}_{1}, \ldots, \mathbb{S}_{L}\right)$ of semisimple $\Lambda$-modules with $\mathbb{S}_{0}=T$ and $\sum_{i} \operatorname{dim} \mathbb{S}_{i}=d$, the subset $\mathfrak{G r a s s}(\mathbb{S})$ of $\mathfrak{G} \mathfrak{r a s s}{ }_{d}^{T}$ is either empty or irreducible of dimension

$$
\text { (multiplicity of } T \text { in } M)-\operatorname{dim} \operatorname{End}_{\Lambda}(M)
$$

for some (equivalently, for all) modules $M$ with radical layering $\mathbb{S}$.

While finite local representation type at $T$ forces $\mathbb{S}(-)$ to separate isomorphism classes, the dimension vector fails to separate in general (Example 5.4).

Notation and terminology. Throughout, we will assume $\Lambda$ to be basic. Due to the algebraic closedness of the ground field $K$, we may thus, without loss of generality, assume that $\Lambda=K Q / I$, where $Q$ is a quiver, and $I$ an admissible ideal in the path algebra $K Q$; the latter means that $I$ is contained in the ideal generated by all paths of length 2 and contains some power of this ideal. The quiver provides us with a convenient set of primitive idempotents $e_{1}, \ldots, e_{n}$ of $\Lambda$, which are in bijective correspondence with the vertices of $Q$; we will, in fact, not distinguish between the vertices and the $e_{i}$. As is well known, the factors $S_{i}=\Lambda e_{i} / J e_{i}$ form a set of representatives of the simple (left) $\Lambda$-modules. By $L$ we denote the largest integer for which the power $J^{L}$ of the Jacobson radical does not vanish; in other words, $L+1$ is the Loewy length of $\Lambda$.

Given any (left) $\Lambda$-module $M$, an element $x \in M$ will be called a top element of $M$ if $x \notin J M$ and $x$ is normed by some $e_{i}$, that is, $x=e_{i} x$ for some $i \in\{1, \ldots, n\}$. The isomorphism invariant

$$
\mathbb{S}(M)=\left(M / J M, J M / J^{2} M, \ldots, J^{L-1} M / J^{L} M, J^{L} M\right)
$$

of $M$ will be referred to as the sequence of radical layers of $M$, or, more briefly, the radical layering of $M$.

Moreover, we observe the following conventions: The product $p q$ of two paths $p$ and $q$ in $K Q$ stands for 'first $q$, then $p$ ' if end $(q)=\operatorname{start}(p)$, and zero otherwise (so, in particular, $p=p e_{i}$ means that the path $p$ starts in the vertex $e_{i}$ ). In line with this notation, we call a path $p_{1}$ a right subpath of $p$ if $p=p_{2} p_{1}$ for some path $p_{2}$. We will generally gloss over the distinction between the left $\Lambda$-structure of $M \in \Lambda$ - mod and the induced left $K Q$-structure; in particular, we let paths operate on $\Lambda$-modules without using residue notation.

For some background on moduli problems, we refer to [16, but recall the definition of a fine moduli space for our specific problem. Our concept of a family of $\Lambda$-modules is that introduced by King in [12, namely, a family of d-dimensional $\Lambda$-modules parametrized by an algebraic variety $X$ is a pair $(\Delta, \delta)$, where $\Delta$ is a (geometric) vector bundle of rank $d$ over $X$ and $\delta: \Lambda \rightarrow \operatorname{End}(\Delta)$ a $K$-algebra homomorphism. Our notion of equivalence of families parametrized by the same variety $X$, finer than King's in general, is the coarsest possible to separate isomorphism classes, namely, $\left(\Delta_{1}, \delta_{1}\right) \sim\left(\Delta_{2}, \delta_{2}\right)$ precisely when, for each $x \in X$, the fibre of $\Delta_{1}$ over $x$ is $\Lambda$-isomorphic to the fibre of $\Delta_{2}$ over $x$. As is common, given a family $(\Delta, \delta)$ parametrized by $X$ and a morphism $\tau: Y \rightarrow X$ of varieties, the induced family $\tau^{*}(\Delta, \delta)$ over $Y$ is the pullback of $(\Delta, \delta)$ along $\tau$. In this context, a variety $X$ is a fine moduli space for (families of) $d$-dimensional modules with top $T$ if there exists a family $(\Gamma, \gamma)$ of such modules parametrized by $X$ which has the property that an arbitrary family - parametrized by $Y$ say - is equivalent to a family $\tau^{*}(\Gamma, \gamma)$ induced via a unique morphism $\tau: Y \rightarrow X$; accordingly $\Gamma$ is called the universal 
family in case of existence. In particular, the requirements on $\Gamma$ entail that every $d$-dimensional module with top $T$ is isomorphic to precisely one fibre of the bundle $\Gamma$. For the more common definition of a fine moduli space through representability of a suitable functor, as well as for the concept of a coarse moduli space, see [16, pp. 23, 24].

\section{The VARIETIEs $\operatorname{Mod}_{d}^{T}$ AND $\mathfrak{G r a s s}_{d}^{T}$ FOr general $T$}

We fix $d \geq 1$ together with a finitely generated semisimple module $T$, say $T=$ $\bigoplus_{1<i<n}\left(\Lambda e_{i} / J e_{i}\right)^{t_{i}}$. Moreover, we let $P=\bigoplus_{1<i<n}\left(\Lambda e_{i}\right)^{t_{i}}$ be the projective cover of $T$. In the first three subsections, we discuss the classical and Grassmannian varieties associated to the $d$-dimensional modules with top $T$, along with connections between them.

2.A. The classical setup. If we denote by $Q^{*}$ the union of the set $\left\{e_{1}, \ldots, e_{n}\right\}$ of vertices with the set of arrows of $Q$, then $Q^{*}$ generates $\Lambda$ as a $K$-algebra, and the traditional variety of $d$-dimensional left $\Lambda$-modules, $\mathbf{M o d}_{d}^{\Lambda}$, takes on the form

$$
\operatorname{Mod}_{d}^{\Lambda}=\left\{\left(x_{\alpha}\right)_{\alpha \in Q^{*}} \in \prod_{\alpha \in Q^{*}} \operatorname{End}_{K}\left(K^{d}\right) \mid\right.
$$

the $x_{\alpha}$ satisfy all the relations of the $\alpha$ in $\Lambda$ \}.

This affine algebraic variety carries a morphic $\mathrm{GL}_{d}$-action by conjugation accounting for change-of-basis transformations. Clearly, the fibres of the representation map

$$
\left.R: \operatorname{Mod}_{d}^{\Lambda} \longrightarrow \text { isomorphism types of } d \text {-dimensional } \Lambda \text {-modules }\right\}
$$

are precisely the $\mathrm{GL}_{d}$-orbits of $\mathbf{M o d}_{d}^{\Lambda}$. The slice of $\mathbf{M o d}_{d}^{\Lambda}$ which will be pivotal for our investigation is the locally closed subvariety

$$
\operatorname{Mod}_{d}^{T}=\operatorname{Mod}_{d}^{\Lambda, T}=\left\{x \in \operatorname{Mod}_{d}^{\Lambda} \mid R(x) / J R(x) \cong T\right\} .
$$

Clearly, $\operatorname{Mod}_{d}^{T}$ is stable under the $\mathrm{GL}_{d}$-action, and its $\mathrm{GL}_{d}$-orbits bijectively parametrize the $d$-dimensional modules with top $T$. Hence, the plausible next step in looking for a moduli space is to attempt factoring the $\mathrm{GL}_{d}$-action out of $\mathbf{M o d}_{d}^{T}$.

We recall two concepts of quotient of an algebraic variety modulo an algebraic group action which will be crucial in the sequel. Suppose that $X$ is a variety endowed with a morphic action of an algebraic group $G$. Then a categorical quotient of $X$ by $G$ is a morphism of varieties $\pi: X \rightarrow Z$ which is constant on the $G$-orbits of $X$ and satisfies the following universal property: every morphism $\psi: X \rightarrow Y$ that is constant on the orbits factors uniquely through $\pi$. Moreover, a morphism $\pi: X \rightarrow Z$ having fibres coinciding with the $G$-orbits is called a geometric quotient of $X$ by $G$ if it is surjective and open and satisfies the following condition relating the structure sheaf of $Z$ to the rings of invariants for the $G$-action: for every open subset $U$ of $Z$, the comorphism $\pi^{*}$ induces an isomorphism from the $\operatorname{ring} \mathcal{O}_{Z}(U)$ of regular functions on $U$ to the $\operatorname{ring} \mathcal{O}_{X}\left(\pi^{-1}(U)\right)^{G}$ of $G$-invariant regular functions on $\pi^{-1}(U)$. Every geometric quotient of $X$ by $G$ is a categorical quotient and hence unique in the case of existence. It is, moreover, well known that, in case $X$ is affine and $G$ reductive (e.g., in case $X=\operatorname{Mod}_{d}^{\Lambda}$ and $G=\mathrm{GL}_{d}$ ), a categorical quotient of $X$ by $G$ always exists; this quotient is geometric precisely when all $G$-orbits of $X$ are closed. Sufficiency of this latter condition does not extend to quasi-affine varieties, however; for counterexamples based on subvarieties of $\mathbf{M o d}_{d}^{\Lambda}$, see Section 4. 
We begin with a well-known criterion characterizing the existence of a coarse moduli space, specialized to a class of modules represented by a subvariety (= locally closed subset) of $\operatorname{Mod}_{d}^{\Lambda}$. It is due to Mumford [15, but we refer to Newstead's formulation in [16], since we have adopted the terminology of that text.

Criterion 2.1. Let $X$ be a $\mathrm{GL}_{d}$-stable subvariety of $\operatorname{Mod}_{d}^{\Lambda}$ and $\mathcal{C}$ the collection of modules represented by $X$. Then there exists a coarse moduli space classifying the modules in $\mathcal{C}$ up to isomorphism if and only if $X$ has a categorical quotient $\pi: X \rightarrow X / \mathrm{GL}_{d}$ which is an orbit map; the latter means that the map $\pi$ is surjective and that its fibres coincide with the $\mathrm{GL}_{d}$-orbits of $X$. In the positive case, the quotient $X / \mathrm{GL}_{d}$ is the coarse moduli space we are looking for.

Proof. By [16, Proposition 2.13], it suffices to specify a family $(\Upsilon, \varepsilon)$ of $\Lambda$-modules, parametrized by $X$, which has the local universal property relative to families of $d$-dimensional modules with top $T$. Local universality means that, for an arbitrary family $(\Delta, \delta)$ - parametrized by some variety $Y$ say - and for any point $y \in Y$, there exists a neighborhood $U$ of $y$ such that $\left.\Delta\right|_{U}$ is induced from $(\Upsilon, \varepsilon)$ by way of some (not necessarily unique) morphism $U \rightarrow X$. The obvious candidate for $(\Upsilon, \varepsilon)$ is as follows: take $\Upsilon$ to be the trivial bundle of rank $d$ over $X$, and define $\varepsilon: \Lambda \rightarrow \operatorname{End}(\Upsilon)$ by the requirement that, for $\alpha \in Q^{*}$, the endomorphism $\varepsilon(\alpha)$ of $\Upsilon$ coincides with $x_{\alpha}$ on the fibre above $x$. To verify the local universal property, it obviously suffices to show that every family $(\Delta, \delta)$ based on a trivial bundle $\Delta=U \times K^{d}$ is induced from $(\Upsilon, \varepsilon)$. To do so, we let $\tau: U \rightarrow X$ be the morphism which sends any element $y$ in $U$ to the point $x \in X$ with the property that, for each $\alpha \in Q^{*}$, the endomorphism $x_{\alpha}$ equals the restriction of $\delta(\alpha)$ to the fibre above $y$. Then $(\Delta, \delta)$ is equivalent to $\tau^{*}(\Upsilon, \varepsilon)$ as required.

A necessary condition for the existence of a coarse moduli space for the $d$ dimensional representations with top $T$ is thus immediate, namely, all $\mathrm{GL}_{d}$-orbits of $\operatorname{Mod}_{d}^{T}$ need to be closed (in standard jargon, this amounts to excluding the 'jump phenomenon'). In representation-theoretic terms, this condition says that no $d$-dimensional module $M$ with top $T$ has a proper degeneration sharing that top.

Recall that a degeneration of a $d$-dimensional module $R(x)$ is a module $R(y)$ with the property that $y$ belongs to the closure of the orbit $\mathrm{GL}_{d} . x$ in $\operatorname{Mod}_{d}^{\Lambda}$. One writes $R(x) \leq_{\operatorname{deg}} R(y)$ in that case to reflect the fact that 'degenerates to' is a partial order on the isomorphism classes of $d$-dimensional modules. For background on degenerations, we point to [4, 13, 17, 21, 2]. In view of Criterion 2.1, our present investigation of moduli spaces automatically involves existence questions for degenerations. As byproducts, we will thus obtain some preliminary results on what we call 'top-stable' and 'layer-stable' degenerations. (This thread will be picked up in [10.)

Definition 2.2. A degeneration $M^{\prime}$ of a finitely generated left $\Lambda$-module $M$ is called top-stable if $M / J M \cong M^{\prime} / J M^{\prime}$, layer-stable in case $J^{l} M / J^{l+1} M \cong J^{l} M^{\prime} / J^{l+1} M^{\prime}$ for all $l$.

Clearly, the layer-stable degenerations of $M$ are a fortiori top-stable. Moreover, it is obvious that the top-stable degenerations of a module $M=R(x)$ with $x \in$ $\operatorname{Mod}_{d}^{T}$ are precisely those $R(y)$ for which $y$ belongs to the relative closure of $\mathrm{GL}_{d} \cdot x$ in $\operatorname{Mod}_{d}^{T}$. Analogously, if $\mathbb{S}=\mathbb{S}(M)$, then the layer-stable degenerations of $M$ 
coincide with the $R(y)$ where $y$ traces the relative closure of $\mathrm{GL}_{d} . x$ in the subvariety

$$
\operatorname{Mod}(\mathbb{S})=\left\{x \in \operatorname{Mod}_{d}^{T} \mid R(x) \text { has radical layering } \mathbb{S}\right\} .
$$

2.B. The Grassmannian setup. Keep the notation introduced at the beginning of Section 2, and set $d^{\prime}=\operatorname{dim}_{K} P-d$. We define $\mathfrak{G r a s s}_{d}^{T}$ to be the closed subvariety of the Grassmannian of $d^{\prime}$-dimensional subspaces of $J P$ consisting of those subspaces that are $\Lambda$-submodules of $J P$. In other words, $\mathfrak{G r a s s}_{d}^{T}$ contains precisely those $\Lambda$-submodules $C$ of $J P$ for which $P / C$ is a $d$-dimensional module (automatically having top $T$ ). Clearly, $\mathfrak{G r a s s}_{d}^{T}$ is a closed subvariety of the usual Grassmannian of $d^{\prime}$-dimensional subspaces of $J P$ and, as such, is in turn projective. Note moreover that the variety $\mathfrak{G r a s s}_{d}^{T}$ comes coupled with a surjective map $\phi=\phi_{d}^{T}: \mathfrak{G r a s s}_{d}^{T} \longrightarrow\{$ isom. types of $d$-diml. modules with top $T\}, \quad C \mapsto[P / C]$.

We will refer to this map as the representation map of the Grassmannian of $d$ dimensional modules with top $T$. As already pointed out, the fibres of $\phi$ coincide with the orbits of the natural morphic action of $\operatorname{Aut}_{\Lambda}(P)$ on $\mathfrak{G r a s s}_{d}^{T}$, denoted by f.C. The fact that the $\operatorname{Aut}_{\Lambda}(P)$-orbits of $\mathfrak{G r a s s}_{d}^{T}$ will turn out to be structurally simpler than the $\mathrm{GL}_{d}$-orbits of $\operatorname{Mod}_{d}^{T}$ in many cases hinges on the following elementary fact.

Observation 2.3. $\operatorname{Aut}_{\Lambda}(P) \cong \operatorname{Aut}_{\Lambda}^{u}(P) \rtimes \operatorname{Aut}_{\Lambda}(T)$, where

$$
\operatorname{Aut}_{\Lambda}^{u}(P)=\left\{\mathrm{id}+f_{0} \mid f_{0} \in \operatorname{Hom}_{\Lambda}(P, J P)\right\}
$$

is the unipotent radical of $\operatorname{Aut}_{\Lambda}(P)$. The automorphism group $\operatorname{Aut}_{\Lambda}(T)$ of the top $T$ of $P$ is isomorphic to $\prod_{1 \leq i \leq n} \mathrm{GL}_{t_{i}}(K)$, a torus if all the $t_{i}$ are at most 1 .

The isomorphism $\operatorname{Aut}_{\Lambda}(\bar{P}) \cong \operatorname{Aut}_{\Lambda}^{u}(P) \rtimes \operatorname{Aut}_{\Lambda}(T)$ can be replaced by equality if, for instance, we identify $\operatorname{Aut}_{\Lambda}(T)$ with the subgroup $\mathcal{H} \leq \operatorname{Aut}_{\Lambda}(P)$ consisting of those $\Lambda$-automorphisms of $P$ which leave the subspace $\bigoplus_{1 \leq i \leq n}\left(K e_{i}\right)^{t_{i}}$ of $P=$ $\bigoplus_{1 \leq i \leq n}\left(\Lambda e_{i}\right)^{t_{i}}$ invariant.

We use hereditary algebras for two simple illustrations: First let $\Lambda=K Q$, where $Q$ is the quiver $2=1$. Then $\mathfrak{G r a s s}_{2}^{S_{1}} \cong \mathfrak{G r a s s}_{3}^{S_{1}} \cong \mathbb{P}^{2}$.

Now suppose that $\Lambda=K Q$, where $Q$ is the quiver 2 $\mathfrak{G r a s s}_{4}^{S_{1}}$ consists of four irreducible components (which coincide with the connected components), two of them copies of $\mathbb{P}^{2} \times \mathbb{P}^{2}$ and the other two singletons. In all of these instances, the $\operatorname{Aut}_{\Lambda}(P)$-orbits are reduced to points; that is, the representation map $\phi$ is a bijection from $\mathfrak{G r a s s}_{4}^{S_{1}}$ to the isomorphism types of 4-dimensional modules with top $S_{1}$.

Yet, in the following example, it is not.

Example 2.4. Let $\Lambda=K Q /\left\langle\omega^{2}\right\rangle$, where $Q$ is the quiver

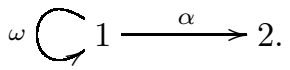

Then $\mathfrak{G r a s s}_{3}^{S_{1}} \cong \mathbb{P}^{1}$, but there are only two isomorphism classes of $d$-dimensional modules with top $S_{1}$, namely, $\Lambda e_{1} / \Lambda(\alpha-k \alpha \omega)$ for $k \in K$ and $\Lambda e_{1} / \Lambda \alpha \omega$.

In general, it is difficult to determine the structure of $\mathfrak{G r a s s}_{d}^{T}$ 'at one blow'. This is one of the reasons for subdividing $\mathfrak{G r a s s}_{d}^{T}$ into more accessible affine portions, still stable under the $\operatorname{Aut}_{\Lambda}(P)$-action. Provided that they are nicely located within 
$\mathfrak{G} \mathfrak{r a s s} \mathfrak{s}_{d}^{T}$, one can then piece together the representation-theoretic information encoded in $\mathfrak{G r a s s}_{d}^{T}$ from the collection of affine frames. Such a subdivision will be introduced in two stages (Sections 2.D and 3.B).

2.C. Connection between $\operatorname{Mod}_{d}^{T}$ and $\mathfrak{G r a s s}_{d}^{T}$. Geometric information on the orbits and orbit closures of the $\operatorname{Aut}_{\Lambda}(P)$-action of $\mathfrak{G r a s s}_{d}^{T}$ smoothly translates into information about the orbits and orbit closures of the $\mathrm{GL}_{d}$-action of the classical variety $\operatorname{Mod}_{d}^{T}$, as spelled out in the following proposition. It is due to Bongartz and the author [6, Proposition $\mathrm{C}$ ] and was previously applied towards an investigation of uniserial modules, which triggered many of the ideas developed here.

Proposition 2.5. Consider the map from the set of all $\operatorname{Aut}_{\Lambda}(P)$-orbits of $\mathfrak{G r a s s}_{d}^{T}$ to the set of $\mathrm{GL}_{d}$-orbits of $\operatorname{Mod}_{d}^{T}$, sending any $\operatorname{Aut}_{\Lambda}(P)$-orbit $\operatorname{Aut}_{\Lambda}(P) . C=$ $\phi^{-1}(P / C)$ to the $\mathrm{GL}_{d}$-orbit $R^{-1}(P / C)$. This map extends to a one-to-one inclusionrespecting correspondence between the set of $\operatorname{Aut}_{\Lambda}(P)$-stable subsets of $\mathfrak{G r a s s}_{d}^{T}$ on the one hand, and the set of $\mathrm{GL}_{d}$-stable subsets of $\operatorname{Mod}_{d}^{T}$ on the other, which preserves openness, closures, irreducibility, connectedness, and types of singularities.

Furthermore, suppose that $V$ is an $\operatorname{Aut}_{\Lambda}(P)$-stable subvariety of $\mathfrak{G r a s s}_{d}^{T}$ and $W$ the corresponding $\mathrm{GL}_{d}$-stable subvariety of $\operatorname{Mod}_{d}^{T}$. Then:

(a) $V$ has a geometric quotient by its $\operatorname{Aut}_{\Lambda}(P)$-action if and only if $W$ has a geometric quotient by its $\mathrm{GL}_{d^{-}}$action, and in the positive case, these quotients coincide.

(b) $V$ has a categorical quotient modulo $\operatorname{Aut}_{\Lambda}(P)$ if and only if $W$ has a categorical quotient modulo $\mathrm{GL}_{d}$. In the positive case, these quotients are isomorphic. The fibres of the former are equal to the $\operatorname{Aut}_{\Lambda}(P)$-orbits of $V$ if and only if the fibres of the latter are equal to the $\mathrm{GL}_{d}$-orbits of $W$.

Proof. Only the statements under (b) were not addressed in [6. They are immediate consequences of Lemmas 1 and 2 preceding [6, Proposition C]. Indeed, a variety $X$ endowed with an action of an algebraic group $H$ having the following properties is presented there: $H$ contains $\operatorname{Aut}_{\Lambda}(P)$ and $\mathrm{GL}_{d}$ as subgroups meeting only in 1 , as well as two closed normal subgroups $N_{1}$ and $N_{2}$ such that $H / N_{1} \cong \operatorname{Aut}_{\Lambda}(P)$ and $H / N_{2} \cong \mathrm{GL}_{d}$ canonically. Moreover, [6] exhibits morphisms $\rho: X \rightarrow \mathfrak{G r a s s}_{d}^{T}$ and $\sigma: X \rightarrow \operatorname{Mod}_{d}^{T}$ with $\phi \circ \rho=R \circ \sigma$ having the following properties: $\rho$ is an

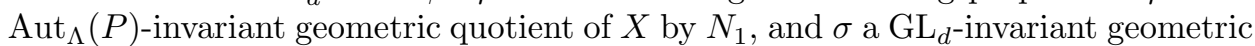
quotient of $X$ by $N_{2}$. It is straightforward to deduce (b).

The final assertion of Proposition 2.5 yields a twin version of Criterion 2.1 which rephrases existence of a coarse moduli space for the $d$-dimensional modules with top $T$ in terms of the $\operatorname{Aut}_{\Lambda}(P)$-space $\mathfrak{G r a s s}_{d}^{T}$.

Criterion 2.6. Let $V$ be any $\operatorname{Aut}_{\Lambda}(P)$-stable subvariety of $\mathfrak{G r a s s}_{d}^{T}$. Then there exists a coarse moduli space for the representations $P / C$ with $C \in V$ if and only if the $\operatorname{Aut}_{\Lambda}(P)$-space $V$ has a categorical quotient which is an orbit map. In the case of existence, such a quotient $V / \operatorname{Aut}_{\Lambda}(P)$ is the moduli space we are looking for.

We also glean an alternate characterization of top-stable degenerations from Proposition 2.5. Observe that, whenever $\phi(C)=P / C \cong R(x)$, the correspondence of the proposition pairs the $\operatorname{Aut}_{\Lambda}(P)$-orbits contained in the closure of $\operatorname{Aut}_{\Lambda}(P) . C$ in $\mathfrak{G r a s s}_{d}^{T}$ with the $\mathrm{GL}_{d}$-orbits contained in the closure of $\mathrm{GL}_{d} . x$ in $\operatorname{Mod}_{d}^{T}$. In 
other words, the top-stable degenerations of $P / C$ are precisely the factors $P / C^{\prime}$ with $C^{\prime} \in \overline{\operatorname{Aut}_{\Lambda}(P) . C}$.

2.D. The partition of $\mathfrak{G r a s s}_{d}^{T}$ in terms of radical layers. In translating geometric features of the variety $\mathfrak{G r a s s}_{d}^{T}$ into representation-theoretic data, the partition of $\mathfrak{G r a s s}_{d}^{T}$ introduced in this section is pivotal. Again, we let the top $T$ equal $\bigoplus_{1 \leq i \leq n}\left(\Lambda e_{i} / J e_{i}\right)^{t_{i}}$ and let $P$ be its projective cover; moreover, we abbreviate the dimension $\sum_{i} t_{i}$ of $T$ by $t$. Furthermore, we will systematically identify isomorphic semisimple modules in the sequel.

Definitions 2.7. (i) A d-dimensional semisimple sequence with top $T$ is a sequence

$$
\mathbb{S}=\left(\mathbb{S}_{0}, \mathbb{S}_{1}, \ldots, \mathbb{S}_{L}\right)
$$

with the following properties: $\mathbb{S}_{0}=T$ and each $\mathbb{S}_{i}$ is a submodule of $J^{i} P / J^{i+1} P$ such that $\sum_{i} \operatorname{dim} \mathbb{S}_{i}=d$.

(ii) Given a $d$-dimensional semisimple sequence $\mathbb{S}=\left(\mathbb{S}_{0}, \ldots, \mathbb{S}_{L}\right)$ with top $T$, we set

$$
\mathfrak{G r a s s}(\mathbb{S})=\left\{C \in \mathfrak{G r a s s}_{d}^{T} \mid \mathbb{S}(P / C)=\mathbb{S}\right\}
$$

and call the $(L+1)$-tuple of integers

$$
\underline{\operatorname{dim}} \mathbb{S}=\left(\operatorname{dim}_{K} \mathbb{S}_{0}, \ldots, \operatorname{dim}_{K} \mathbb{S}_{L}\right)
$$

the dimension vector of $\mathbb{S}$.

Since we are identifying isomorphic semisimple modules, there are only finitely many $d$-dimensional semisimple sequences. The important examples are the radical layerings of the $d$-dimensional modules with top $T$; indeed, whenever $C \in \mathfrak{G r a s s}_{d}^{T}$, the sequence $\mathbb{S}(P / C)$ - see end of Section 1 - meets our criteria.

We start by recording the 'layer-stable analogue' of the final observation of 2.C. Namely, again by Proposition 2.5, the layer-stable degenerations of a module $P / C$ with $C \in \mathfrak{G} \mathfrak{r a s s}(\mathbb{S})$ are precisely the modules represented by the points in the relative closure of $\operatorname{Aut}_{\Lambda}(P) . C$ in $\mathfrak{G r a s s}(\mathbb{S})$.

It is, moreover, obvious that each of the sets $\mathfrak{G} \mathfrak{r a s s}(\mathbb{S})$ is stable under the $\operatorname{Aut}_{\Lambda}(P)$-action and that $\mathfrak{G r a s s}_{d}^{T}$ is the disjoint union of the $\mathfrak{G r a s s}(\mathbb{S})$, where $\mathbb{S}$ ranges through the $d$-dimensional semisimple sequences with top $T$. We point out that, in general, this partition falls slightly short of being a stratification in the technical sense: while the sets $\mathfrak{G} \mathfrak{r a s s}(\mathbb{S})$ are locally closed subvarieties of $\mathfrak{G r a s s}_{d}^{T}$ - see part (ii) of the upcoming lemma - their closures are not always unions of $\mathfrak{G r a s s}\left(\mathbb{S}^{\prime}\right)$ 's. However, this lack is compensated for by the following asset (see part (i) of the lemma). Endow the $(L+1)$-tuples of nonnegative integers with the lexicographic order, and let $\mathbf{d} \in \mathbb{N}_{0}^{L+1}$. Then the union $\bigcup_{\underline{\text { dim }} \mathbb{S}>\mathbf{d}} \mathfrak{G r a s s}(\mathbb{S})$, where $\mathbb{S}$ runs through the $d$-dimensional semisimple sequences with top $T$, is closed in $\mathfrak{G r a s s}_{d}^{T}$.

We can do a little better than that by introducing the following partial order on the semisimple sequences themselves. Namely, for two $d$-dimensional semisimple sequences $\mathbb{S}=\left(\mathbb{S}_{0}, \ldots, \mathbb{S}_{L}\right)$ and $\mathbb{S}^{\prime}=\left(\mathbb{S}_{0}^{\prime}, \ldots, \mathbb{S}_{L}^{\prime}\right)$, we define

$$
\mathbb{S} \leq \mathbb{S}^{\prime}
$$

if and only if $\bigoplus_{0 \leq i \leq L} \mathbb{S}_{i}=\bigoplus_{0 \leq i \leq L} \mathbb{S}_{i}^{\prime}$ and either $\mathbb{S}=\mathbb{S}^{\prime}$ or else $\mathbb{S}_{j}$ is a proper direct summand of $\mathbb{S}_{j}^{\prime}$ for the smallest index $j$ with $\mathbb{S}_{j} \neq \mathbb{S}_{j}^{\prime}$. Clearly, this partial order is compatible with the order on the dimension vectors, in the sense that $\mathbb{S} \leq \mathbb{S}^{\prime}$ implies $\underline{\operatorname{dim}} \mathbb{S} \leq \underline{\operatorname{dim}} \mathbb{S}^{\prime}$. 
Lemma 2.8. (i) Let $\mathbb{S}$ be any d-dimensional semisimple sequence with top $T$. Then $\bigcup_{\mathbb{S}^{\prime} \leq \mathbb{S}} \mathfrak{G r a s s}\left(\mathbb{S}^{\prime}\right)$ is open in $\mathfrak{G r a s s}_{d}^{T}$, as is the union $\bigcup_{\mathbb{S}^{\prime}<\mathbb{S}} \mathfrak{G r a s s}\left(\mathbb{S}^{\prime}\right)$. Consequently, the sets $\bigcup_{\text {dim } \mathbb{S} \geq \mathbf{d}} \mathfrak{G r a s s}(\mathbb{S})$ and $\bigcup_{\underline{\operatorname{dim}} \mathbb{S}>\mathbf{d}} \mathfrak{G r a s s}(\mathbb{S})$ are closed for any $(L+1)$-tuple d of nonnegative integers.

(ii) Each of the sets $\mathfrak{G r a s s}(\mathbb{S})$ is locally closed in $\mathfrak{G r a s s}_{d}^{T}$.

(iii) If $M$ is a d-dimensional module with top $T$ and $M^{\prime}$ a top-stable degeneration of $M$, then $\underline{\operatorname{dim}} \mathbb{S}(M) \leq \underline{\operatorname{dim}} \mathbb{S}\left(M^{\prime}\right)$.

Proof. We fix a sequence $z_{1}, \ldots, z_{t}$ of top elements of $P$, where each $z_{r}$ is normed by a primitive idempotent, $e(r)$ say, such that $P=\bigoplus_{1<r<t} \Lambda z_{r}$.

(i) Let $\mathcal{B}$ be a subset of the projective $K Q$-module $\bigoplus_{1 \leq r \leq t} K Q e(r)$ consisting of elements of the form $p \mathbf{e}(r)$, where $p$ is a path starting in the vertex $e(r)$, and $\mathbf{e}(r)$ is the element of the direct sum carrying the idempotent $e(r)$ in the $r$-th slot and 0 elsewhere. We say that such a set $\mathcal{B}$ of "labeled paths" is admissible if $\mathcal{B}$ includes the candidates $\mathbf{e}(1), \ldots, \mathbf{e}(r)$ of length zero and $|\mathcal{B}|=d$. Moreover, given a $d$-dimensional semisimple sequence $\mathbb{S}$ with top $T$, we call an admissible set $\mathcal{B}$ compatible with $\mathbb{S}$ in case, for each $l \in\{0, \ldots, L\}$ and each $i \in\{1, \ldots, n\}$, the number of paths of length $l$ in $\mathcal{B}$ ending in $e_{i}$ equals the multiplicity of the corresponding simple module $S_{i}$ in $\mathbb{S}_{l}$ (for $l=0$, this is automatic). As before, $d^{\prime}=\operatorname{dim} P-d$. For every admissible set $\mathcal{B}$, finally, we let $\operatorname{Schu}(\mathcal{B})$ be the set of all points $C$ in the classical Grassmannian $\operatorname{Gr}\left(d^{\prime}, J P\right)$ of $d^{\prime}$-dimensional subspaces of $J P$ with the property that

$$
C \oplus\left(\sum_{r \leq t} \sum_{p \mathbf{e}(r) \in \mathcal{B}} K p z_{r}\right)=P
$$

here we identify the paths $p$ with their residue classes modulo $I$. If $\operatorname{Schu}(\mathcal{B})$ is nonempty, then $\operatorname{Schu}(\mathcal{B})$ is a Schubert cell of $\operatorname{Gr}\left(d^{\prime}, J P\right)$, and the open subset $\operatorname{Schu}(\mathcal{B}) \cap \mathfrak{G} \mathfrak{r a s s}_{d}^{T}$ of $\mathfrak{G r a s s}_{d}^{T}$ consists precisely of those $d^{\prime}$-dimensional $\Lambda$-submodules $C \subseteq J P$ for which $P / C$ has basis $\bigcup_{1 \leq r \leq t}\left\{p z_{r}+C \mid p \mathbf{e}(r) \in \mathcal{B}\right\}$.

To verify the openness of $\bigcup_{\mathbb{S}^{\prime}<\mathbb{S}} \mathfrak{G r a s s}\left(\mathbb{S}^{\prime}\right)$, it now suffices to notice that $\bigcup_{\mathbb{S}^{\prime}<\mathbb{S}} \mathfrak{G r a s s}\left(\mathbb{S}^{\prime}\right)$ is the union of the intersections $\operatorname{Schu}(\mathcal{B}) \cap \mathfrak{G r a s s}_{d}^{T}$, where $\mathcal{B}$ traces the admissible sets that are compatible with some $\mathbb{S}^{\prime}$ smaller than $\mathbb{S}$. The inclusion of the latter union in the former follows from the fact that, whenever $\mathcal{B}$ is compatible with $\mathbb{S}^{\prime}$ and $C \in \operatorname{Schu}(\mathcal{B}) \cap \mathfrak{G r a s s}_{d}^{T}$, the module $P / C$ has radical layering $\mathbb{S}(P / C) \leq \mathbb{S}^{\prime}$; the other inclusion is obvious. Analogously, $\bigcup_{\mathbb{S}^{\prime} \leq \mathbb{S}} \mathfrak{G r a s s}\left(\mathbb{S}^{\prime}\right)$ is seen to be open.

(ii) This follows from (i) and the fact that $\mathfrak{G} \mathfrak{r a s s}(\mathbb{S})$ is the intersection of $\bigcup_{\mathbb{S}^{\prime} \leq \mathbb{S}} \mathfrak{G r a s s}\left(\mathbb{S}^{\prime}\right)$ with $\bigcup_{\mathbb{S}^{\prime} \nless \mathbb{S}} \mathfrak{G r a s s}\left(\mathbb{S}^{\prime}\right)$.

(iii) It suffices to observe that the relative closure of $\mathfrak{G r a s s}(\mathbb{S}(M))$ in $\mathfrak{G r a s s}_{d}^{T}$ is contained in $\bigcup_{\underline{\operatorname{dim}} \mathbb{S} \geq \underline{\operatorname{dim}} \mathbb{S}(M)} \mathfrak{G r a s s}(\mathbb{S})$.

2.E. General results on the $\operatorname{Aut}_{\Lambda}(P)$-orbits. The following proposition summarizes the information on the $\operatorname{Aut}_{\Lambda}(P)$-orbits of $\mathfrak{G r a s s}_{d}^{T}$ - alias the fibres of the representation map $\phi$-available without any restriction on $T$. Strengthened versions for the squarefree case will come to bear in Section 4.

Again, we let $T=\bigoplus_{1 \leq i \leq n}\left(\Lambda e_{i} / J e_{i}\right)^{t_{i}}$, set $t=\sum_{i} t_{i}$, and fix a sequence $z_{1}, \ldots, z_{t}$ of top elements of $P$, each $z_{r}$ being normed by a primitive idempotent $e(r)$, such that $P=\bigoplus_{1 \leq r \leq t} \Lambda z_{r}$. If $\mathcal{H}$ is the subgroup of $\operatorname{Aut}_{\Lambda}(P)$ consisting of all automorphisms which leave the subspace $\bigoplus_{1 \leq r \leq t} K z_{i}$ invariant, and $\mathcal{U}$ the unipotent radical of 
$\operatorname{Aut}_{\Lambda}(P)$, then Observation 2.3 tells us that $\mathcal{U}$ is normal in $\operatorname{Aut}_{\Lambda}(P)$ and $\operatorname{Aut}_{\Lambda}(P)$ is a semidirect product $\mathcal{U} \rtimes \mathcal{H}$. The incarnation of the maximal torus of $\mathcal{H}$ afforded by our choice of top elements of $P$ is $\mathcal{T}=\left(K^{*}\right)^{t}$, where we identify $\left(a_{1}, \ldots, a_{t}\right) \in \mathcal{T}$ with the automorphism of $P$ given by $z_{i} \mapsto a_{i} z_{i}$. Finally, given $h \in \mathcal{H}$ and $C \in$ $\mathfrak{G r a s s}_{d}^{T}$, we denote by $h . \mathcal{U}$.C the set $\{h u . C \mid u \in \mathcal{U}\}$. Due to the normality of $\mathcal{U}$ in $\operatorname{Aut}_{\Lambda}(P)$, the set h.U.C equals the $\mathcal{U}$-orbit of h.C.

Proposition 2.9. Let $C \in \mathfrak{G r a s s}_{d}^{T}$.

(1) The orbit $\operatorname{Aut}_{\Lambda}(P) . C$ has dimension

$$
\operatorname{dim}_{K} \operatorname{End}_{\Lambda}(P)-\operatorname{dim}_{K} \operatorname{Hom}_{\Lambda}(P, C)-\operatorname{dim}_{K} \operatorname{End}_{\Lambda}(P / C),
$$

and this dimension is generically constant on the irreducible components of $\mathfrak{G r a s s}_{d}^{T}$.

(2) The $\mathcal{U}$-orbit $\mathcal{U}$.C in $\mathfrak{G r a s s}_{d}^{T}$ is isomorphic to $\mathbb{A}^{m}$ with

$$
m=m(P / C)=\operatorname{dim}_{K} \operatorname{Hom}_{\Lambda}(P, J P / C)-\operatorname{dim}_{K} \operatorname{Hom}_{\Lambda}(P / C, J P / C) .
$$

(3) There exists a point $C^{\prime} \in \operatorname{Aut}_{\Lambda}(P) . C$ such that the $\mathcal{T}$-orbit $\mathcal{T}$. $C^{\prime}$ is isomorphic to the torus $\left(K^{*}\right)^{t-\mathfrak{s}}$, where $\mathfrak{s}=\mathfrak{s}(P / C)$ is the number of indecomposable summands of $P / C$.

(4) The full orbit $\operatorname{Aut}_{\Lambda}(P) . C$ is the disjoint union of the subvarieties h.U.C. $\cong$ $\mathbb{A}^{m(P / C)}$, for $h \in \mathcal{H}$, and $\mathcal{H}$ acts as a transitive permutation group on these subvarieties.

Before proving the proposition, we single out a lemma for repeated reference.

Lemma 2.10. For any $C \in \mathfrak{G r a s s}_{d}^{T}$, the orbit map $\operatorname{Aut}_{\Lambda}(P) \rightarrow \operatorname{Aut}_{\Lambda}(P) . C$, $f \mapsto f . C$, is separable, and hence $\operatorname{Aut}_{\Lambda}(P) . C$ is isomorphic to the homogeneous $\operatorname{Aut}_{\Lambda}(P)$-space $\operatorname{Aut}_{\Lambda}(P) / \operatorname{Stab}_{\operatorname{Aut}_{\Lambda}(P)} C$. Moreover, the dimensions of the stabilizers of $C$ in $\operatorname{Aut}_{\Lambda}(P)$, resp. in $\mathcal{U}$, are:

$$
\operatorname{dim} \operatorname{Stab}_{\operatorname{Aut}_{\Lambda}(P)} C=\operatorname{dim}_{K} \operatorname{Hom}_{\Lambda}(P, C)+\operatorname{dim}_{K} \operatorname{End}_{\Lambda}(P / C)
$$

and

$$
\operatorname{dim} \operatorname{Stab}_{\mathcal{U}} C=\operatorname{dim}_{K} \operatorname{Hom}_{\Lambda}(P, C)+\operatorname{dim}_{K} \operatorname{Hom}_{\Lambda}(P / C, J P / C) .
$$

Proof. We first address the separability claim. Combining [7, Proposition 6.7] with [13. AI.5.5, Satz 2], we see that it suffices to check reducedness of the schematic fibre of the orbit map, i.e., reducedness of the stabilizer subgroup $\operatorname{Stab}_{\operatorname{Aut}_{\Lambda}(P)} C$ of $C$. Reducedness of this stabilizer, in turn, can be deduced from the fact that it arises as the solution set of a system of linear equations over $K$; ascertaining the latter fact is a matter of routine, if notationally cumbersome.

For the first dimension formula, consider the homomorphism $\rho: \operatorname{Stab}_{\operatorname{Aut}_{\Lambda}(P)} C \rightarrow$ $\operatorname{Aut}_{\Lambda}(P / C)$ of algebraic groups sending any map $f$ in the stabilizer of $C$ to the induced automorphism of $P / C$. Clearly, $\rho$ is onto and the kernel is the subgroup of $\operatorname{Aut}_{\Lambda}(P)$ consisting of the automorphisms of the form id $+g_{0}$ with $g_{0} \in$ $\operatorname{Hom}_{\Lambda}(P, C)$. This proves the first equality.

As for the second, consider the restriction of $\rho$ to $\operatorname{Stab}_{\mathcal{U}} C$, and notice that the kernel is the same as before, while $\rho\left(\mathrm{Stab}_{\mathcal{U}} C\right)$ equals the unipotent radical of $\operatorname{Aut}_{\Lambda}(P / C)$, namely the subgroup consisting of the automorphisms of the form id $+g_{1}$ with $g_{1} \in \operatorname{Hom}_{\Lambda}(P / C, J P / C)$. 
Proof of Proposition 2.9. (1) Since

$$
\operatorname{dim} \operatorname{Aut}_{\Lambda}(P) \cdot C=\operatorname{dim} \operatorname{Aut}_{\Lambda}(P)-\operatorname{dim} \operatorname{Stab}_{\operatorname{Aut}_{\Lambda}(P)} C,
$$

the claimed equality can be derived from Lemma 2.10. For the generic behavior of the fibre dimension, see, e.g., [13, II.2.6].

(2) In view of Lemma 2.10, U.C is isomorphic to the homogeneous space $\mathcal{U} / \operatorname{Stab}_{\mathcal{U}} C$, whence the claim concerning the fibre structure follows from Rosenlicht's Theorem [19]. The assertion concerning the fibre dimension is a consequence of the equality $\operatorname{dim} \mathcal{U}=\operatorname{dim}_{K} \operatorname{Hom}_{\Lambda}(P, J P)$ and Lemma 2.10.

(3) Clearly, $\mathcal{T} . C \cong \mathcal{T} / \operatorname{Stab}_{\mathcal{T}} C$ is a torus. For the following choice of $C^{\prime} \in$ $\operatorname{Aut}_{\Lambda}(P) . C$ we will show that $\operatorname{dim} \operatorname{Stab}_{\mathcal{T}} C^{\prime}=\mathfrak{s}$. Decompose $P / C$ into indecomposable summands, say $P / C \cong P_{1} / C_{1}^{\prime} \oplus \cdots \oplus P_{\mathfrak{s}} / C_{\mathfrak{s}}^{\prime}$, where the $P_{i}$ are suitable direct summands of $P$; it is clearly harmless to assume $P_{i}=\bigoplus_{j \in I(i)} \Lambda z_{j}$ for some partition $\bigsqcup_{1 \leq i \leq \mathfrak{s}} I(i)$ of $\{1, \ldots, t\}$. Now set $C^{\prime}=\bigoplus_{1 \leq i \leq \mathfrak{s}} C_{i}^{\prime}$. For simplicity of notation, we assume $C^{\prime}=C$. Let $\widehat{\mathcal{T}}$ be the $K$-subspace of $\operatorname{End}_{\Lambda}(P)$ consisting of all endomorphisms of the form $\left(a_{1}, \ldots, a_{t}\right) \in K^{t}$, not just the invertible ones; again we identify $\left(a_{1}, \ldots, a_{t}\right)$ with the endomorphism of $P$ sending $z_{i}$ to $a_{i} z_{i}$. Moreover, $\operatorname{Stab}_{\hat{\mathcal{T}}} C$ will stand for the $K$-subspace of $\widehat{\mathcal{T}}$ consisting of the endomorphisms $f$ with $f(C) \subseteq C$. Clearly, $\operatorname{Stab}_{\mathcal{T}} C$ is a dense open subvariety of $\operatorname{Stab}_{\hat{\mathcal{T}}} C$, whence the two varieties have the same dimension. As for the dimension of the latter, $\operatorname{Stab}_{\hat{\mathcal{T}}} C$ obviously contains the span of the vectors $\mathbf{1}_{I(i)}$, defined as having $j$-th entry 1 if $j \in I(i)$ and 0 otherwise. That these vectors in fact form a basis for $\mathrm{Stab}_{\hat{\mathcal{T}}} C$ is an immediate consequence of the indecomposability of the $P_{i} / C_{i}$. We infer that $\operatorname{Stab}_{\mathcal{T}} C$ has dimension $\mathfrak{s}$, which makes the dimension of $\mathcal{T}$. $C$ equal to $t-\mathfrak{s}$ as asserted.

(4) is obvious in view of the remarks preceding the proposition.

As part (3) of the proposition suggests, the dimension of the stabilizer subgroup $\operatorname{Stab}_{\mathcal{T}} C^{\prime}$ varies from one point $C^{\prime} \in \operatorname{Aut}_{\Lambda}(P) . C$ to another. In fact, $\operatorname{Stab}_{\mathcal{T}} C^{\prime}$ may be reduced to the scalar multiples of the identity $\mathbf{1}$ of $\mathcal{T}$, even when $\mathfrak{s}(P / C)$ is large.

\section{3. $\mathfrak{G r a s s}_{d}^{T}$ FOR SQUAREFREE $T$}

The diagram below is to remind the reader of the relationships among the varieties in which we are interested.

$\underline{\text { Classical scenario } \quad \text { Grassmannian scenario }}$

$$
\operatorname{Mod}_{d}^{T} \stackrel{R}{\mathrm{GL}_{d} \text {-action }} \longrightarrow\left\{\begin{array}{c}
\text { isom. types of } \\
d \text {-dim'l. modules } \\
\text { with top } T
\end{array}\right\} \longleftrightarrow \operatorname{Aut}_{\Lambda}(P) \text {-action }
$$

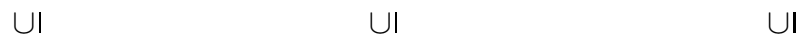

$$
\operatorname{Mod}(\mathbb{S}) \stackrel{R}{\longrightarrow}\left\{\begin{array}{c}
\text { isom. types of } \\
\text { modules with } \\
\text { radical layering } \mathbb{S}
\end{array}\right\} \longleftarrow \phi(\mathfrak{G} \mathfrak{r a s s}(\mathbb{S})
$$


Throughout this section, $T$ denotes a squarefree semisimple module, say

$$
T=\bigoplus_{1 \leq r \leq t} \Lambda e_{r} / J e_{r}
$$

and $P=\bigoplus_{1 \leq r \leq t} \Lambda e_{r}$ its projective cover; in particular, $P$ is now a left ideal of $\Lambda$. We again set $d^{\prime}=\operatorname{dim}_{K} P-d$. Our primary goal is to construct finite $\operatorname{Aut}_{\Lambda}(P)$ stable affine open covers of the subvarieties $\mathfrak{G} \mathfrak{r a s s}(\mathbb{S}) \subseteq \mathfrak{G r a s s}_{d}^{T}$.

3.A. Convenient local coordinates for $\mathfrak{G r a s s}_{d}^{T}$ and a pivotal family of representations parametrized by $\mathfrak{G r a s s}_{d}^{T}$. We could have postponed the restriction to squarefree $T$ until the next section, but adopt it here for its notational pay-off. The coordinatized open cover of $\mathfrak{G r a s s}_{d}^{T}$, which we introduce first, does not consist of $\operatorname{Aut}_{\Lambda}(P)$-stable sets in general. It depends on a fixed choice of top elements of $P$, whereas the cover at which we are ultimately aiming is $\operatorname{Aut}_{\Lambda}(P)$-stable and invariant under such choices. At the outset, our preferred bases for factor modules $P / C$ may appear more specialized than necessary for the present purpose. They actually are; yet, it is precisely this special format which will make them useful in the sequel.

The second definition below is merely of temporary importance as an auxiliary to the proofs.

Definitions 3.1. (i) A d-dimensional skeleton with top $T$ is a set $\sigma$ that consists of $d$ distinct paths of lengths $\leq L$ in $K Q$ and satisfies the following conditions:

- each path in $\sigma$ starts in one of the vertices $e_{1}, \ldots, e_{t}$;

- $\sigma$ is closed under right subpaths;

- $\sigma$ contains $\left\{e_{1}, \ldots, e_{t}\right\}$.

(ii) Given a $d$-dimensional skeleton $\sigma$ with top $T$, we define the auxiliary set

$$
\operatorname{Aux}(\sigma)=\left\{C \in \mathfrak{G} \mathfrak{r a s s}_{d}^{T} \mid P / C \text { has basis }\{p+C \mid p \in \sigma\}\right\} .
$$

A $d$-dimensional skeleton $\sigma$ with top $T$ can be visualized as an undirected graph with $d$ nodes, namely the disjoint union of $t$ trees, represented by the sets $\left\{p \in \sigma \mid p=p e_{r}\right\}$ for $1 \leq r \leq t$. Clearly, there are only finitely many $d$-dimensional skeletons. Moreover, $\operatorname{Aux}(\sigma)$ is empty unless the subset $\sigma$ of $P$ is linearly independent over $K$. On the other hand, each point $C \in \mathfrak{G r a s s}_{d}^{T}$ belongs to at least one of the sets $\operatorname{Aux}(\sigma)$; in other words, the $\operatorname{Aux}(\sigma)$ cover $\mathfrak{G r a s s}_{d}^{T}$; see Lemma 3.9 for a sharper statement.

By definition, the sets $\operatorname{Aux}(\sigma)$ are intersections of $\mathfrak{G r a s s}_{d}^{T}$ with open Schubert cells in the full Grassmannian $\operatorname{Gr}\left(d^{\prime}, J P\right)$ in which $\mathfrak{G r a s s}_{d}^{T}$ is located. Namely, for any skeleton $\sigma$, the relevant open cell is

$$
\left\{C \in \operatorname{Gr}\left(d^{\prime}, J P\right) \mid C \oplus\left(\sum_{\substack{p \in \sigma \\ \operatorname{length}(p) \geq 1}} K p\right)=J P\right\} .
$$

Observation 3.2. The $\operatorname{Aux}(\sigma)$ are open subsets of $\mathfrak{G r a s s}_{d}^{T}$.

Next, we will use this cover to construct a family of $d$-dimensional modules with top $T$ parametrized by $\mathfrak{G} \mathfrak{r a s s} \mathfrak{s}_{d}^{T}$. The reason why this family is of interest lies in the fact that it melts down to the universal family whenever a fine moduli space for our problem exists. 
Lemma 3.3. There exists a family $(\Gamma, \gamma)$ of d-dimensional modules parametrized by $\mathfrak{G r a s s}_{d}^{T}$ such that, for each $C \in \mathfrak{G}_{\mathfrak{r a s s}}^{T}$, the fibre of $\Gamma$ over $C$ (with the $\Lambda$-structure induced by $\gamma$ ) is isomorphic to $P / C$.

Proof. Let $\operatorname{Mod}_{d}^{\Lambda}$ be the affine variety of Section 2.A and $\sigma$ a $d$-dimensional skeleton with top $T$. We start by pinning down the obvious morphism $\rho_{\sigma}$ from $\operatorname{Aux}(\sigma)$ to $\operatorname{Mod}_{d}^{\Lambda}$ which matches our coordinatization. To that end, we assume that $\operatorname{Aux}(\sigma) \neq$ $\varnothing$. This implies that the $K$-subspace $V$ of $P$ generated by $\sigma$ has dimension $d$ and, given any $C \in \operatorname{Aux}(\sigma)$, each element of $P$ has a unique expansion as a $K$-linear combination of the elements $p \in \sigma$ modulo $C$. View $V$ as $K^{d}$, identifying the distinguished basis $\sigma$ of $V$ with the canonical basis of $K^{d}$, where $\sigma$ is ordered as follows. Start by ordering the finite set $\mathcal{P}$ of all paths of length $\leq L$ in $K Q$ in such a fashion that the paths starting in $e_{i}$ precede those starting in $e_{j}$ whenever $i<j$. Then let $p_{1}$ be that element of $\sigma$ which is minimal in $\mathcal{P}$, next let $p_{2} \in \sigma$ be as small as possible in $\mathcal{P} \backslash\left\{p_{1}\right\}$, etc.

Given $C \in \operatorname{Aux}(\sigma)$, we now define

$$
\rho_{\sigma}(C)=\left(\rho_{\sigma}(C)_{\alpha}\right)_{\alpha \in Q^{*}},
$$

where $\rho_{\sigma}(C)_{\alpha}$ is the $d \times d$ matrix over $K$ recording in its columns the expansion coefficients (relative to $\sigma$ ) of the multiples $\alpha p$ modulo $C$, for $p \in \sigma$.

Thus the trivial bundle $\operatorname{Aux}(\sigma) \times K^{d}$ becomes a family of $\Lambda$-modules via the algebra homomorphism from $\Lambda$ to the endomorphism ring of $\operatorname{Aux}(\sigma) \times K^{d}$, defined by

$$
\alpha \mapsto\left((C, v) \mapsto\left(C, \rho_{\sigma}(C)_{\alpha} v\right)\right)
$$

The appropriate choice of morphisms $g_{\sigma, \sigma^{\prime}}: \operatorname{Aux}(\sigma) \cap \operatorname{Aux}\left(\sigma^{\prime}\right) \rightarrow \mathrm{GL}_{d}$ to glue the $\operatorname{Aux}(\sigma) \times K^{d}$ together to a vector bundle over $\mathfrak{G r a s s}_{d}^{T}$ is now automatic. Namely, just send each $C \in \operatorname{Aux}(\sigma) \cap \operatorname{Aux}\left(\sigma^{\prime}\right)$ to the transition matrix recording the coefficients of the $p^{\prime}+C$ (for $p^{\prime} \in \sigma^{\prime}$ ) relative to the $p+C$, in the prescribed order; then, clearly, the $g_{\sigma, \sigma^{\prime}}$ satisfy the relevant cocycle condition. The resulting vector bundle over $\mathfrak{G r a s s}_{d}^{T}$ will be denoted by $\Gamma$. Since our algebra homomorphisms $\Lambda \rightarrow \operatorname{End}\left(\operatorname{Aux}(\sigma) \times K^{d}\right)$ are compatible with this gluing, they yield an algebra homomorphism $\gamma: \Lambda \rightarrow \operatorname{End}(\Gamma)$ and thus a family of $d$-dimensional $\Lambda$-modules as required.

3.B. The distinguished $\operatorname{Aut}_{\Lambda}(P)$-stable affine open cover of $\mathfrak{G r a s s}(\mathbb{S})$. The key to the announced cover comes from the following counterparts of the skeletons of section 3.A. By construction, this new cover will depend only on the quiver of $\Lambda$ and the ideal $I$ of relations. In fact, up to permutation and birational equivalence of the irreducible components, it is an isomorphism invariant of $\Lambda$ (see Section 3.18).

Definitions 3.4. (i) Let $M$ be a $d$-dimensional module with top $T$. A $d$-dimensional skeleton $\sigma$ (with top $T$ ) is called a skeleton of $M$ provided there exists a sequence $x_{1}, \ldots, x_{t}$ of top elements of $M$ with $x_{r}=e_{r} x_{r}$ such that, for every integer $l$ between 0 and $L$, the union

$$
\bigcup_{1 \leq r \leq t}\left\{p x_{r}+J^{l+1} M \mid p=p e_{r} \in \sigma, \operatorname{length}(p)=l\right\}
$$

is a $K$-basis for $J^{l} M / J^{l+1} M$.

(ii) For any $d$-dimensional skeleton $\sigma$ with top $T$, we set

$$
\mathfrak{G r a s s}(\sigma)=\left\{C \in \mathfrak{G r a s s}_{d}^{T} \mid \sigma \text { is a skeleton of } P / C\right\} .
$$


(iii) Given a skeleton $\sigma$ and a semisimple sequence $\mathbb{S}$, we say that $\sigma$ is compatible with $\mathbb{S}$ in case, for each $i \in\{1, \ldots, n\}$, the number of paths of any prescribed length $l$ in $\sigma$ which end in the vertex $e_{i}$ equals $\operatorname{dim}_{K} e_{i} \mathbb{S}_{l}$.

First we observe that the notion of a skeleton of a module $M$ as in (i) does not depend on a choice of top elements of $M$. Indeed, as is readily confirmed, if $\sigma$ is a skeleton of $M$, then any choice of top elements $y_{1}=e_{1} y_{1}, \ldots, y_{t}=e_{t} y_{t}$ of $M$ yields $K$-bases $\bigcup_{1 \leq r \leq t}\left\{p y_{r}+J^{l+1} M \mid p=p e_{r} \in \sigma\right.$, length $\left.(p)=l\right\}$ for the radical layers $J^{l} M / J^{l+1} M$.

It is obvious that isomorphic modules have identical sets of skeletons, which means that the sets $\mathfrak{G} \mathfrak{r a s s}(\sigma)$ are stable under the action of $\operatorname{Aut}_{\Lambda}(P)$. Moreover, we notice that, whenever $C$ belongs to $\mathfrak{G} \mathfrak{r a s s}(\sigma)$, the skeleton $\sigma$ is compatible with the sequence $\mathbb{S}(P / C)$ of radical layers of $P / C$. Hence $\mathfrak{G} \mathfrak{r a s s}(\sigma) \cap \mathfrak{G} \mathfrak{r a s s}(\mathbb{S})=\varnothing$ if $\sigma$ fails to be compatible with $\mathbb{S}$. On the other hand, $\mathfrak{G} \mathfrak{r a s s}(\sigma)$ is contained in $\mathfrak{G r a s s}(\mathbb{S})$, whenever $\sigma$ is compatible with $\mathbb{S}$. Further properties of the sets $\mathfrak{G} \mathfrak{r a s s}(\sigma)$ are collected in the following theorem. In particular, we glean from it that the $\mathfrak{G r a s s}(\sigma)$ are locally closed affine subvarieties of $\mathfrak{G} \mathfrak{r a s s}_{d}^{T}$.

Theorem 3.5. Let $\mathbb{S}$ be a d-dimensional semisimple sequence with top $T$.

The sets $\mathfrak{G} \mathfrak{r a s s}(\sigma)$, where $\sigma$ runs through the skeletons compatible with $\mathbb{S}$, form a finite $\operatorname{Aut}_{\Lambda}(P)$-stable affine open cover of the quasi-projective variety $\mathfrak{G} \mathfrak{r a s s}(\mathbb{S})$. A family of polynomials determining any of the $\mathfrak{G} \mathfrak{r a s s}(\sigma)$ in a suitable affine coordinate system can be (algorithmically) obtained from the quiver $Q$ and a finite set of relations generating the ideal $I$ as a left ideal of $K Q$.

The fact that the sets $\mathfrak{G} \mathfrak{r a s s}(\sigma)$ form an open cover of $\mathfrak{G} \mathfrak{r a s s}(\mathbb{S})$ will be proved in Lemmas 3.8 and 3.9; we emphasize that the $\mathfrak{G} \mathfrak{r a s s}(\sigma)$ fail to be open in $\mathfrak{G r a s s}_{d}^{T}$ though. The remaining claim of Theorem 3.5, namely that each of the sets $\mathfrak{G} \mathfrak{r a s s}(\sigma)$ is an affine variety that can be obtained from the quiver and the relations of $\Lambda$, is less obvious. We will tackle it in several steps, providing further details along the way.

Remark 3.6. The affine cover of Theorem 3.5 permits us to resolve the isomorphism problem for $d$-dimensional top- $T$ modules with low computational investment: given any point $C$ in $\mathfrak{G} \mathfrak{r a s s}(\sigma)$, expressed in terms of an affine coordinate system for $\mathfrak{G} \mathfrak{r a s s}(\sigma)$, the $\operatorname{Aut}_{\Lambda}(P)$-orbit of $C$ can be obtained from a system of at most $d \cdot\left(\operatorname{dim}_{K} J / J^{2}\right)$ linear equations [unpublished notes].

The following elementary example shows that, if $T$ contains squares of simples, the variety $\mathfrak{G} \mathfrak{r a s s}(\mathbb{S})$ will not have an $\operatorname{Aut}_{\Lambda}(P)$-stable affine cover in general. In fact, we exhibit an $\operatorname{Aut}_{\Lambda}(P)$-orbit that fails to be quasi-affine for a situation where $T$ is a square.

Example 3.7. Let $\Lambda$ be the representation-finite hereditary algebra $K Q$, where $Q$ is the quiver

$$
2 \longleftarrow \alpha
$$

If $\mathbb{S}=\left(S_{1}^{2}, S_{2} \oplus S_{3}\right)$, then $\mathfrak{G r a s s}(\mathbb{S})$ is isomorphic to $\mathbb{P}^{1} \times \mathbb{P}^{1}$ and consists of two $\operatorname{Aut}_{\Lambda}(P)$-orbits, namely the orbits representing the modules $\Lambda e_{1} / \Lambda \alpha e_{1} \oplus \Lambda e_{1} / \Lambda \beta e_{1}$ and $\Lambda e_{1} \oplus S_{1}$. The orbit of the latter is isomorphic to $\mathbb{P}^{1}$. 
We return to our blanket hypothesis that $T$ be squarefree. In justifying Theorem 3.5 , we start with openness of the $\mathfrak{G} \mathfrak{r a s s}(\sigma)$ in the pertinent $\mathfrak{G} \mathfrak{r a s s}(\mathbb{S})$.

Lemma 3.8. For any skeleton $\sigma$ compatible with $\mathbb{S}$, the subset $\mathfrak{G} \mathfrak{r a s s}(\sigma)$ is open in $\mathfrak{G} \mathfrak{r a s s}(\mathbb{S})$.

Proof. From Definitions 3.1 and 3.4 one readily deduces that, under our compatibility hypothesis,

$$
\mathfrak{G r a s s}(\sigma)=\operatorname{Aux}(\sigma) \cap \mathfrak{G r a s s}(\mathbb{S}) .
$$

Observation 3.2 thus proves our claim.

That the $\mathfrak{G} \mathfrak{r a s s}(\sigma)$ cover $\mathfrak{G} \mathfrak{r a s s}(\mathbb{S})$ is guaranteed by the following elementary lemma.

Lemma 3.9. Every module $M$ with top $T$ has at least one skeleton.

Proof. First, we set $\sigma_{0}=\left\{e_{1}, \ldots, e_{t}\right\}$ and choose top elements $x_{1}=e_{1} x_{1}, \ldots, x_{t}=$ $e_{t} x_{t}$ of $M$ giving rise to a basis of $M / J M$. Next, we pick a set $\sigma_{1}$ of arrows $p_{1}$, each starting in one of the vertices in $\sigma_{0}$, such that the set $\left\{p_{1} x_{r} \mid p_{1} \in \sigma_{1} \cap K Q e_{r}, 1 \leq\right.$ $r \leq t\}$ yields a $K$-basis for $J M$ modulo $J^{2} M$. The following stage is to choose a set $\sigma_{2}$ of paths $p_{2}$ of length 2, each containing some arrow in $\sigma_{1}$ as a right subpath, such that the set $\left\{p_{2} x_{r} \mid p_{2} \in \sigma_{2} \cap K Q e_{r}, 1 \leq r \leq t\right\}$ gives rise to a $K$-basis of $J^{2} M / J^{3} M$. We continue in this fashion and set $\sigma=\bigcup_{0 \leq l \leq L} \sigma_{l}$. (Keep in mind that $J^{L+1}=0$ by our choice of $L$.)

From the proof of Lemma 3.9, we can actually glean the following stronger statement: given any path $p$ of length $l$ with $p M \nsubseteq J^{l+1} M$, there exists a skeleton of $M$ which contains $p$.

We next introduce a convenient affine coordinate grid for each $\mathfrak{G r a s s}(\sigma)$, and then follow with a family of polynomials derived from the relations of $\Lambda$. That the vanishing set of these polynomials coincides with $\mathfrak{G} \mathfrak{r a s s}(\sigma)$, up to an isomorphism which 'respects' the representation map, is spelled out under the heading of the more precise Theorem 3.14 below.

3.10. The coordinate system for $\mathfrak{G} \mathfrak{r a s s}(\sigma)$. Let $\sigma$ be a $d$-dimensional skeleton with top $T$. As we know from 3.A, $\mathfrak{G} \mathfrak{r a s s}(\sigma)$ embeds into the intersection $\operatorname{Aux}(\sigma)$ of $\mathfrak{G r a s s}_{d}^{T}$ with the open Schubert cell consisting of those subspaces of $J P$ which have zero intersection with the span of $\sigma$ (see Definitions 3.1). In essence, the coordinates with which we will work are Plücker coordinates relative to an ordered basis of $J P$ which supplements the paths of positive length in $\sigma$ to a $K$-basis of $J P$ made up of paths. However, our situation permits restriction to a small subfamily of these coordinates, determined by paths of the form $\alpha p$, where $(\alpha, p)$ is a $\sigma$-critical pair as defined below. As pointed out in the introduction, this economy is crucial in the analysis of concrete examples; polynomial equations governing these pared-down coordinates can be read off from the quiver and the relations of $\Lambda$.

A pair $(\alpha, p)$, consisting of an arrow $\alpha$ and a path $p \in \sigma$, is called $\sigma$-critical in case $\alpha p$ is a path in $K Q$ which does not belong to $\sigma$.

Clearly, the isomorphism type of any module $M=P / C$ with skeleton $\sigma$ is completely pinned down by the (unique) scalars $c_{\alpha p, q}$ appearing in the following equations in $M$ :

$$
\alpha p+C=\sum_{q \in \sigma} c_{\alpha p, q} q+C
$$


for the $\sigma$-critical pairs $(\alpha, p)$. Moreover, to obtain a more economical coordinatization, we observe that the coefficient $c_{\alpha p, q}$ in the above equation is zero unless $q$ is strictly longer than $p$, and has the same starting and ending vertices as $\alpha p$. This motivates the following notation: for any $\sigma$-critical pair $(\alpha, p)$, we let $\sigma(\alpha, p)$ be the set of all paths in $\sigma$ which are at least as long as $\alpha p$ and have the same end vertex as $\alpha p$.

As a consequence, in pinning down $M$, we only need to keep track of the scalars arising in the equations

$$
\alpha p+C=\sum_{q \in \sigma(\alpha, p)} c_{\alpha p, q} q+C
$$

for the $\sigma$-critical pairs $(\alpha, p)$. In other words, as a $\Lambda$-submodule of $P$, any $C \in$ $\mathfrak{G} \mathfrak{r a s s}(\sigma)$ is generated by the differences

$$
\alpha p-\sum_{q \in \sigma(\alpha, p)} c_{\alpha p, q} q .
$$

As long as we keep the skeleton $\sigma$ fixed, it is therefore justified to identify the points $C \in \mathfrak{G} \mathfrak{r a s s}(\sigma)$ with the corresponding families

$$
\left(c_{\alpha p, q}\right)_{\substack{(\alpha, p) \sigma-\text { critical } \\ q \in \sigma(\alpha, p)}}
$$

of scalars in the appropriate affine space $\mathbb{A}^{N}$, where $N$ is the (artificially) disjoint union of the sets $\sigma(\alpha, p)$.

3.11. The congruence relation induced by $\sigma$. Keeping $\sigma$ fixed, we consider the polynomial ring

$$
\mathcal{A}=\mathcal{A}(\sigma):=K Q\left[X_{\alpha p, q} \mid(\alpha, p) \sigma \text {-critical, } q \in \sigma(\alpha, p)\right]
$$

over the path algebra $K Q$. On the ring $\mathcal{A}$, we consider congruence modulo the left ideal

$$
\mathcal{C}=\mathcal{C}(\sigma):=\bigoplus_{t+1 \leq i \leq n} \mathcal{A} e_{i}+\sum_{(\alpha, p)} \mathcal{A}\left(\alpha p-\sum_{q \in \sigma(\alpha, p)} X_{\alpha p, q} q\right)
$$

and denote this congruence relation by $\widehat{=}$.

The argument backing the following proposition is constructive.

Proposition 3.12. The quotient $\mathcal{A} / \mathcal{C}$ is a free left module over the commutative polynomial ring $K\left[X_{\alpha p, q}\right]$, with the cosets of the paths in $\sigma$ forming a basis.

In other words, for every $z \in \mathcal{A}$, there is a unique family of polynomials $\tau_{q}(X)=$ $\tau_{q}^{z}(X)$ in $K\left[X_{\alpha p, q}\right]$ such that

$$
z \widehat{=} \sum_{q \in \sigma} \tau_{q}(X) q .
$$

Proof. We only verify existence of the $\tau_{q}(X)$ and leave uniqueness to the reader. It is clearly innocuous to assume that $z$ is a path in $K Q$ starting at one of the vertices $e_{1}, \ldots, e_{t}$. Let $p$ be the longest right subpath of $z$ belonging to $\sigma$. We will prove our claim by induction on length $(z)-\operatorname{length}(p)$. If this difference is zero, we are done. So suppose it is positive and let $\alpha$ be the (unique) arrow with the property 
that $\alpha p$ is again a right subpath of $z$, say $z=z^{\prime} \alpha p$ for a suitable path $z^{\prime}$. Then $(\alpha, p)$ is a $\sigma$-critical pair, and

$$
z \widehat{=} z^{\prime}\left(\sum_{q \in \sigma(\alpha, p)} X_{\alpha p, q} q\right)=\sum_{q \in \sigma(\alpha, p)} X_{\alpha p, q} z^{\prime} q .
$$

Since all of the differences length $\left(z^{\prime} q\right)-\operatorname{length}(q)$ arising from this equation are strictly smaller than length $(z)-\operatorname{length}(p)$, our induction hypothesis guarantees that each of the summands $X_{\alpha p, q} z^{\prime} q$ has the desired form up to $\widehat{=}$. Consequently, so does $z$.

3.13. Polynomials for $\mathfrak{G} \mathfrak{r a s s}(\sigma)$. Let $\mathcal{I}(\sigma)$ be the ideal of the polynomial ring $\mathcal{A}(\sigma)$ generated by the ideal $I$ of relations of $\Lambda$, i.e., $I(\sigma)=I\left[X_{\alpha p, q}\right]$. Moreover, let $\mathrm{K}(\sigma)$ be the kernel of the $K\left[X_{\alpha p, q}\right]$-resolution

$$
\left(K\left[X_{\alpha p, q}\right]\right)^{\sigma} \rightarrow \mathcal{A}(\sigma) /(\mathcal{I}(\sigma)+\mathcal{C}(\sigma)),
$$

sending $\left(\tau_{q}(X)\right)_{q \in \sigma}$ to $\sum_{q \in \sigma} \tau_{q}(X) \bar{q}$; here the cyclic left $\mathcal{A}(\sigma)$-module $\mathcal{A}(\sigma) /(\mathcal{I}(\sigma)+\mathcal{C}(\sigma))$ carries the inherited $K\left[X_{\alpha p, q}\right]$-structure. Then, as Theorem 3.14 will show, the variety $\mathfrak{G} \mathfrak{r a s s}(\sigma)$ is isomorphic to the vanishing set in $\mathbb{A}^{N}=\mathbb{A}^{N(\sigma)}$ of the ideal $\sum_{q \in \sigma} \pi_{q}(\mathrm{~K}(\sigma))$ in the commutative polynomial ring $K\left[X_{\alpha p, q}\right]$, where the $\pi_{q}$ for $q \in \sigma$ are the canonical projections of $\left(K\left[X_{\alpha p, q}\right]\right)^{\sigma}$.

Proposition 3.12 allows us to rephrase this in the following leaner terms: Let $\mathcal{R}$ be any finite generating set for the left ideal $I e_{1}+\cdots+I e_{t}$ of $K Q-$ note that such a generating set always exists since all paths of lengths $\geq L+1$ belong to $I$. For each $\rho \in \mathcal{R}$, let $\tau_{q}^{\rho}(X)$ be the unique polynomials in $K\left[X_{\alpha p, q}\right]$ with

$$
\rho \widehat{=} \sum_{q \in \sigma} \tau_{q}^{\rho}(X) q
$$

as guaranteed by Proposition 3.12. Then the locus $V\left(\tau_{q}^{\rho}(X) \mid \rho \in \mathcal{R}, q \in \sigma\right)$ of these polynomials is an alternate incarnation of $\mathfrak{G} \mathfrak{r a s s}(\sigma)$, as we will see.

Solely for the purpose of establishing a suitable isomorphism of varieties, we let $\operatorname{Aff}(\sigma)$ stand for the subvariety of $\mathbb{A}^{N}$ defined by the polynomials $\tau_{q}^{\rho}(X)$ for the moment.

It is straightforward that $\operatorname{Aff}(\sigma)$ is independent of our choice of $\mathcal{R}$. (In fact, so is the ideal of $K\left[X_{\alpha p, q}\right]$ generated by the $\tau_{q}^{\rho}(X)$.) Furthermore, we emphasize that, in order to proceed, we need not preselect 'eligible' $d$-dimensional skeletons, i.e., skeletons which actually occur as skeletons of $\Lambda$-modules; $\operatorname{Aff}(\sigma)$ is automatically empty if $\mathfrak{G} \mathfrak{r a s s}(\sigma)$ is, as is for instance the case when, for some integer $l$, the set of paths of length $l$ in $\sigma$ is linearly dependent modulo $J^{l+1} P$.

Theorem 3.14. Suppose $\sigma$ is a d-dimensional skeleton. There exists an isomorphism $\psi_{\sigma}: \operatorname{Aff}(\sigma) \rightarrow \mathfrak{G r a s s}(\sigma)$ of varieties, together with a surjective map $\chi_{\sigma}$, such that the following diagram commutes:

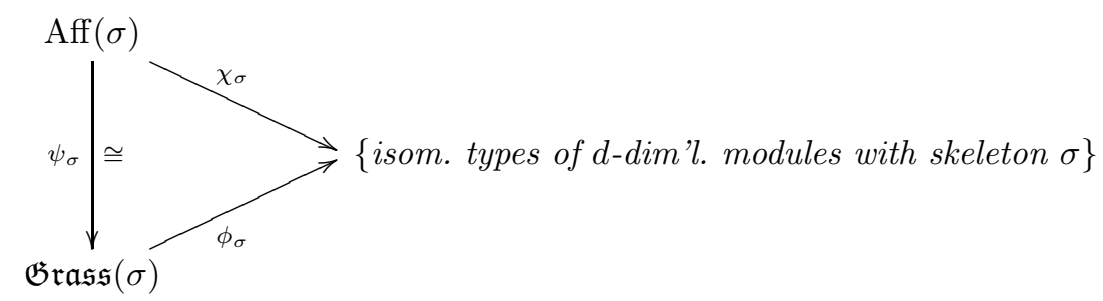


Here $\phi_{\sigma}$ is the restriction of the representation map $\phi$ to $\mathfrak{G} \mathfrak{r a s s}(\sigma)$, and the maps

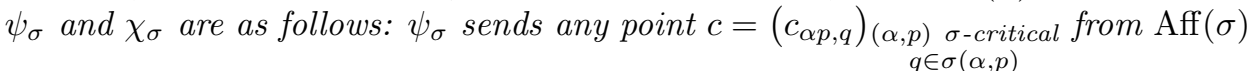
to the submodule

$$
U(c):=\sum_{(\alpha, p)} \Lambda\left(\alpha p-\sum_{\sigma \in \sigma(\alpha, p)} c_{\alpha p, q} q\right)
$$

of $P=\bigoplus_{1 \leq r \leq t} \Lambda e_{r}$, and $\chi_{\sigma}$ sends c to the isomorphism type of the factor module $P / U(c)$.

We defer the somewhat technical proof of Theorem 3.14 to Section 6; clearly, Theorem 3.14 covers the remaining claims of Theorem 3.5.

In the sequel, we will identify $\operatorname{Aff}(\sigma)$ with $\mathfrak{G} \mathfrak{r a s s}(\sigma)$ and $\chi_{\sigma}$ with $\phi_{\sigma}$. This is warranted in light of the commutative diagram linking the pertinent representation maps from $\mathfrak{G} \mathfrak{r a s s}(\sigma)$ and $\operatorname{Aff}(\sigma)$ to the modules with skeleton $\sigma$. We remark that $\psi_{\sigma}^{-1}$ is a closed immersion of $\mathfrak{G} \mathfrak{r a s s}(\sigma)$ into the affine space $\mathbb{A}^{N}$.

An algorithm for finding polynomials that determine $\mathfrak{G} \mathfrak{r a s s}(\sigma)$ within the affine space $\mathbb{A}^{N}$ is implicit in the preceding discussion, but will not be put on a systematic basis here. We will only illustrate the procedure with an example. The following observation significantly reduces the computational effort; we include it, since we will require the underlying concept of a 'route' in the proof of Theorem 3.14.

3.15. A crucial property of the left ideal $\mathcal{C}(\sigma)$ of 3.11. Suppose $u$ is a path of length $l$ which passes through the vertices $(e(0), e(1), \ldots, e(l))$ in that order; in particular, this means that $u$ starts in $e(0)$ and ends in $e(l)$. Given a $d$-dimensional skeleton $\sigma$ with top $T$, such a path $u$ will be called a route on $\sigma$ if there exist paths $p_{0}, p_{1}, \ldots, p_{l}$ in $\sigma$ with length $\left(p_{0}\right)=0$ and length $\left(p_{i-1}\right)<\operatorname{length}\left(p_{i}\right)$ such that each $p_{i}$ ends in $e(i)$. Note that any path in $\sigma$ is a route on $\sigma$, since skeletons are closed under right subpaths by definition.

If $u$ is a path which fails to be a route on $\sigma$, then $u \in \mathcal{C}(\sigma)$; in other words, $u \widehat{=} 0$ under the congruence relation of Section 3.11. Consequently, $u$ can be ignored in the substitution process leading to polynomials defining $\mathfrak{G} \mathfrak{r a s s}(\sigma)$.

To justify this claim, let $C$ be any point in $\mathfrak{G} \mathfrak{r a s s}(\sigma)$, and suppose $u=\alpha_{s} \cdots \alpha_{1}$ is a path, concatenated from arrows $\alpha_{i}$, such that $u+C$ is nonzero in $M=P / C$. If, for $j \leq s$, the integer $l_{j}$ is such that $\alpha_{j} \cdots \alpha_{1}+C \in J^{l_{j}} M \backslash J^{l_{j}+1} M$, then $1<l_{1}<l_{2}<\cdots<l_{s}$; moreover, for each $j$, the set $\sigma$ contains a path of length $l_{j}$ ending in the same vertex as $\alpha_{j}$. This shows the path $u=\alpha_{s} \cdots \alpha_{1}$ to be a route on $\sigma$, thus backing our remark.

Example 3.16. Consider $\Lambda=K Q / I$, where $Q$ is the quiver

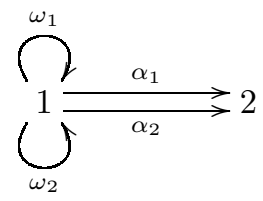

and $I \subseteq K Q$ the ideal generated by the four paths $\omega_{i} \omega_{j}$ for $i, j \in\{1,2\}$ and $\alpha_{1} \omega_{1}-\alpha_{2} \omega_{2}$. Clearly, the listed relations also generate $I e_{1}$ as a left ideal of $K Q$.

For $T=S_{1}$ and $d=4$, we consider the skeleton $\sigma=\left\{e_{1}, \omega_{1}, \alpha_{1} \omega_{1}, \alpha_{2}\right\}$ and determine $\mathfrak{G} \mathfrak{r a s s}(\sigma)$ as follows. First we list the $\sigma$-critical pairs $(\alpha, p)$, together 
with their sets $\sigma(\alpha, p)$. Obviously, there is no harm in omitting pairs $(\alpha, p)$ with $\alpha p \in I$; the pairs that are left are $\left(\omega_{2}, e_{1}\right)$ with $\sigma\left(\omega_{2}, e_{1}\right)=\left\{\omega_{1}\right\},\left(\alpha_{1}, e_{1}\right)$ with $\sigma\left(\alpha_{1}, e_{1}\right)=\left\{\alpha_{2}, \alpha_{1} \omega_{1}\right\}$, and $\left(\alpha_{2}, \omega_{1}\right)$ with $\sigma\left(\alpha_{2}, \omega_{1}\right)=\left\{\alpha_{1} \omega_{1}\right\}$. This leads to the following congruences in $K Q\left[X_{1}, \ldots, X_{4}\right]$, where $X_{1}, \ldots, X_{4}$ stand for $X_{\omega_{2}, \omega_{1}}$, $X_{\alpha_{1}, \alpha_{2}}, X_{\alpha_{1}, \alpha_{1} \omega_{1}}, X_{\alpha_{2} \omega_{1}, \alpha_{1} \omega_{1}}$. Namely, $\omega_{2} \widehat{=} X_{1} \omega_{1}, \alpha_{1} \widehat{=} X_{2} \alpha_{2}+X_{3} \alpha_{1} \omega_{1}$, and $\alpha_{2} \omega_{1} \widehat{=} X_{4} \alpha_{1} \omega_{1}$. Since all of the four paths $\omega_{i} \omega_{j}$ in $I$ fail to be routes on $\sigma$, Remark 3.15 tells us that $\omega_{i} \omega_{j} \widehat{=} 0$ is automatic, whence the relations $\omega_{i} \omega_{j}=0$ in $\Lambda$ do not produce any conditions on the $X_{k}$. On the other hand, inserting the above 'basic congruences' into the final relation yields $\alpha_{1} \omega_{1}-\alpha_{2} \omega_{2} \widehat{=} \alpha_{1} \omega_{1}-X_{1} \alpha_{2} \omega_{1} \widehat{=}$ $\alpha_{1} \omega_{1}-X_{1} X_{4} \alpha_{1} \omega_{1}=\left(1-X_{1} X_{4}\right) \alpha_{1} \omega_{1}$. The last congruence is the expansion of $\alpha_{1} \omega_{1}-\alpha_{2} \omega_{2} \in \mathcal{A}$ as in Proposition 3.12, whence

$$
\mathfrak{G r a s s}(\sigma)=\left\{\left(c_{i}\right)_{i \leq 4} \in \mathcal{A}^{4} \mid 1-c_{1} c_{4}=0\right\} .
$$

3.17. Functoriality of the $\operatorname{Aut}_{\Lambda}(P)$-stable affine charts. Fix the quiver $Q$, and identify $T$ with the corresponding set of vertices $\left\{e_{1}, \ldots, e_{t}\right\}$. Moreover, let $\mathcal{I}$ be the category of all admissible ideals of the path algebra $K Q$ - the morphisms in this category are the inclusion maps - and define $\mathcal{M}$ to be the category of left modules over the ring $\prod_{\sigma} \mathcal{A}(\sigma)$, where $\sigma$ traces the skeletons with top $T$. Now define $F$ to be the functor $\mathcal{I} \rightarrow \mathcal{M}$, which sends any ideal $I$ in $\mathcal{I}$ to the left module $\prod_{\sigma} \mathcal{A}(\sigma) /(I(\sigma)+\mathcal{C}(\sigma))$ (cf. Sections 3.11 and 3.13). By the preceding theorem, the functor $F$ then carries full information about the representation theory with top $\{1 \ldots, t\}$ of arbitrary basic $K$-algebras with quiver $Q$. Indeed, according to Section 3.13 and Theorem 3.14, each image under $F$ determines a family $(\mathfrak{G r a s s}(I, \sigma))_{\sigma}$, where $\mathfrak{G} \mathfrak{r a s s}(I, \sigma)$ denotes the affine chart in $\mathbb{A}^{N(\sigma)}$ which represents the $K Q / I$ modules with top $T$ and skeleton $\sigma$. Finally, if $\operatorname{Var}^{T}$ stands for the category consisting of all families of affine varieties over $K$ indexed by the skeletons $\sigma$ with top $T$ (the maps in this latter category are the corresponding families of morphisms), then the assignment $(I(\sigma))_{\sigma} \mapsto(\mathfrak{G r a s s}(I, \sigma))_{\sigma}$ defines a contravariant functor from $\mathcal{I}$ to $\operatorname{Var}^{T}$ which reverses inclusions.

3.18. Uniqueness of the $\operatorname{Aut}_{\Lambda}(P)$-stable affine charts. Clearly, the locally closed subsets $\mathfrak{G r a s s}(\mathbb{S})$ of $\mathfrak{G r a s s}_{d}^{T}$ are uniquely determined, up to isomorphism, by the isomorphism type of $\Lambda$. The affine open subsets $\mathfrak{G} \mathfrak{r a s s}(\sigma)$ of any given $\mathfrak{G r a s s}(\mathbb{S})$, in their turn, enjoy a somewhat weaker uniqueness property. Namely, they are invariant under algebra isomorphisms of $\Lambda$, up to birational equivalence. More precisely, suppose $\Lambda \cong \Lambda^{\prime}$, where $\Lambda^{\prime}=K Q^{\prime} / I^{\prime}$ (it is well known that $Q$ and $Q^{\prime}$ are isomorphic as directed graphs in this situation). Let $\mathbb{S}$ be a semisimple sequence over $\Lambda$, and $\mathbb{S}^{\prime}$ the semisimple sequence over $\Lambda^{\prime}$ corresponding to $\mathbb{S}$ under some algebra isomorphism from $\Lambda$ to $\Lambda^{\prime}$. Moreover, let $\operatorname{Irr}(\mathbb{S})$ be the set of all irreducible components of the affine varieties $\mathfrak{G} \mathfrak{r a s s}(\sigma)$, where $\sigma$ runs through the skeletons compatible with $\mathbb{S}$, and define $\operatorname{Irr}\left(\mathbb{S}^{\prime}\right)$ analogously. Then there is a bijection $\operatorname{Irr}(\mathbb{S}) \rightarrow$ $\operatorname{Irr}\left(\mathbb{S}^{\prime}\right)$ such that the partners under this pairing are birationally equivalent. One proves this as in the scenario of varieties of uniserial modules in [5. Finally, one observes that 'birationally equivalent' can be strengthened to 'isomorphic' in our uniqueness statement, provided that $Q$ is without double arrows; indeed, in that case, both the quiver and the corresponding relations are essentially pinned down by the isomorphism type of $\Lambda$. 


\section{The MODULi PROBLEM}

We continue to assume that $T=\bigoplus_{1<r<t} \Lambda e_{r} / J e_{r}$ is squarefree, and $P=$ $\bigoplus_{1 \leq r \leq t} \Lambda e_{r}$. The first subsection contains our main results, while in 4 . B we briefly discuss the moduli problem for classes of representations with fixed radical layering.

4.A. Top-stable degenerations and quotients of $\mathfrak{G r a s s}_{d}^{T}$ modulo $\operatorname{Aut}_{\Lambda}(P)$. From Proposition 2.9 and Theorem 3.5, we derive the following consequences concerning top-stable and layer-stable degenerations of modules with squarefree tops.

Proposition 4.1. (1) All $\operatorname{Aut}_{\Lambda}(P)$-orbits are quasi-affine subvarieties of $\mathfrak{G r a s s}_{d}^{T}$.

(2) Whenever $M$ is a module with top $T$, say $M=P / C$ with $C \in \mathfrak{G} \mathfrak{r a s s}(\mathbb{S})$, the orbit $\mathcal{U}$.C of $C$ under the action of the unipotent radical $\mathcal{U}$ of $\operatorname{Aut}_{\Lambda}(P)$ is closed in $\mathfrak{G} \mathfrak{r a s s}(\mathbb{S})$. It is an affine space $\mathbb{A}^{m}$ of dimension

$$
\begin{aligned}
m=m(M) & =\mu_{T}(M)-\left(t+\operatorname{dim}_{K} \operatorname{Hom}_{\Lambda}(M, J M)\right) \\
& =\operatorname{dim}_{K} \operatorname{Hom}_{\Lambda}(P, J M)-\operatorname{dim}_{K} \operatorname{Hom}_{\Lambda}(M, J M),
\end{aligned}
$$

where $\mu_{T}(M)$ is the sum of the multiplicities of the simple summands of $T$ as composition factors of $M$.

(3) In case $T$ is simple and $M$ is as in part (2), the orbit $\operatorname{Aut}_{\Lambda}(P) . C$ is closed in $\mathfrak{G} \mathfrak{r a s s}(\mathbb{S})$ and isomorphic to $\mathbb{A}^{m}$ with

$$
m=\mu_{T}(M)-\operatorname{dim}_{K} \operatorname{End}_{\Lambda}(M),
$$

where $\mu_{\mathcal{T}}(M)$ is the multiplicity of $T$ in $M$.

Remark. In [10], it will be shown that, for squarefree $T$, all $\operatorname{Aut}_{\Lambda}(P)$-orbits of $\mathfrak{G}_{\mathfrak{r a s s}}{ }_{d}^{T}$ are direct products of affine spaces and tori as follows: $\operatorname{Aut}_{\Lambda}(P) . C \cong$ $\mathbb{A}^{m} \times\left(K^{*}\right)^{r}$, where $m$ is as under (2) and $r$ equals the difference $\operatorname{dim} T$ minus the number of indecomposable summands of $M$.

Proof of Proposition 4.1. The first assertion is an immediate consequence of the existence of an $\operatorname{Aut}_{\Lambda}(P)$-stable affine cover of $\mathfrak{G r a s s}_{d}^{T}$ (Theorem 3.5). As for the second, let $C \in \mathfrak{G} \mathfrak{r a s s}(\mathbb{S})$. To see that the $\mathcal{U}$-orbit of $C$ is closed in $\mathfrak{G} \mathfrak{r a s s}(\mathbb{S})$, we need only make sure that its intersections with the open subvarieties $\mathfrak{G r a s s}(\sigma)$, where $\sigma$ runs through the skeletons compatible with $\mathbb{S}$, are relatively closed. But in light of Theorem 3.5, this follows from a result of Kostant and Rosenlicht which guarantees that the orbits of a unipotent group morphically acting on an affine variety are closed (cf. [18, Theorem 2], and [11, p.115, Exercise 8] for a simple alternate proof). The claimed dimension of $\mathcal{U} . C$ can be deduced from Proposition 2.9. Finally for part (3), observe that $\operatorname{Aut}_{\Lambda}(P) \cong K^{*} \times \mathcal{U}$, with $K^{*}$ acting trivially, and apply part (2).

A module is called local in case it has a simple top; thus the local modules are characterized by their having precisely one maximal submodule.

Theorem 4.2. Suppose that $M$ is a d-dimensional module with top $T$, say $M \cong$ $P / C$ with $C \in \mathfrak{G r a s s}_{d}^{T}$. Then the following conditions are equivalent:

(1) $M$ has no proper top-stable degenerations.

(2) The orbit $\operatorname{Aut}_{\Lambda}(P) . C$ is closed in $\mathfrak{G r a s s}_{d}^{T}$.

(3) The orbit $\operatorname{Aut}_{\Lambda}(P) . C$ is a singleton.

(4) $C \subseteq P$ is invariant under $\Lambda$-endomorphisms of $P$, i.e., $C u \subseteq C$ for any path $u$ starting in one of the vertices $e_{r}$ with $r \leq t$. 
(5) $M$ is a direct sum of local modules and

$$
\mu_{\mathcal{T}}(M)=t+\operatorname{dim}_{K} \operatorname{Hom}_{\Lambda}(M, J M),
$$

where $\mu_{T}(M)$ is the sum of the multiplicities of the simple summands of $T$ as composition factors of $M$.

Proof. The equivalence of (1) and (2) is immediate from Proposition 2.5, and the implications $(4) \Longrightarrow(3) \Longrightarrow(2)$ are trivial.

To verify $(2) \Longrightarrow(3)$, suppose (2) holds. Using the fact that $\mathfrak{G r a s s}_{d}^{T}$ is a projective variety, we infer completeness of $\operatorname{Aut}_{\Lambda}(P) . C$. On the other hand, this orbit is quasi-affine by Proposition 4.1, and therefore must be zero dimensional. Since $\operatorname{Aut}_{\Lambda}(P) . C$ is irreducible, (3) follows.

For $(3) \Longrightarrow(4)$, assume (3), which means that $C$ is invariant under automorphisms of $P$. Given any $f \in \operatorname{End}_{\Lambda}(P)$, pick a nonzero scalar $a$ such that $-a$ is not an eigenvalue of $f$. Then $a \cdot \mathrm{id}+f$ belongs to $\operatorname{Aut}_{\Lambda}(P)$ and hence leaves $C$ invariant; but this means $f(C) \subseteq C$.

$(3) \Longrightarrow(5)$. By Proposition 2.9(3), the number of summands in a decomposition of $M$ into indecomposables equals $t$. Since $t=\operatorname{dim}_{K} T$, this means that $M$ splits into local summands. The numerical equality follows from Proposition 4.1(2).

$(5) \Longrightarrow(3)$. Without loss of generality, we may assume that $C$ equals the point $C^{\prime} \in \operatorname{Aut}_{\Lambda}(P) . C$ specified in Proposition 2.9(3). Thus $\mathcal{T} . C=\{C\}$. Moreover, (5) guarantees that $\mathcal{U} . C \cong \mathbb{A}^{0}$, where $\mathcal{U}$ again denotes the unipotent radical of $\operatorname{Aut}_{\Lambda}(P)$. Hence $\operatorname{Aut}_{\Lambda}(P) . C$ is a singleton.

In case $T$ contains squares, the statements of the theorem fail in general. For instance, in Example 3.7, let $M=\Lambda e_{1} \oplus S_{1}$. Then $M=P / C$ with $C=\Lambda(0, \alpha)+$ $\Lambda(0, \beta)$ has no proper top-stable degeneration, while $\operatorname{Aut}_{\Lambda}(P) . C$ is the projective line.

The simplest instance of a (local) module $M$ having a proper top-stable degeneration can be found in Example 2.4. In this example, $\mathfrak{G r a s s}_{d}^{T}$ consists of two $\operatorname{Aut}_{\Lambda}(P)$-orbits, those of the modules $M=\Lambda e_{1} / \Lambda \alpha$ and $M^{\prime}=\Lambda e_{1} / \Lambda \alpha \omega$. The $\operatorname{Aut}_{\Lambda}(P)$-orbit of $C=\Lambda \alpha$ is isomorphic to $\mathbb{A}^{1}$, while that of $C^{\prime}=\Lambda \alpha \omega$ is a singleton. Thus $M$ degenerates top-stably to $M^{\prime}$. However, $M^{\prime}$ is not a layer-stable degeneration of $M$, as is predicted by the following consequence of Proposition 4.1(3).

Corollary 4.3. If $M$ is a $\Lambda$-module with a simple top, then $M$ has no proper layer-stable degenerations.

In contrast, modules with nonsimple tops do have proper layer-stable degenerations in general (see 10).

From Corollary 4.3, it is intuitively clear that the absence of proper layer-stable degenerations of the modules represented by $\operatorname{Mod}(\mathbb{S})$ does not suffice to guarantee existence of a moduli space for the representations with radical layering $\mathbb{S}$; a confirmation will follow in 4.B. The next theorem attributes special properties to $\operatorname{Mod}_{d}^{T}$ and its closed subvarieties. (Recall that $R$ denotes the representation map from $\operatorname{Mod}_{d}^{T}$ to the set of isomorphism classes of $d$-dimensional modules with top T.)

Theorem 4.4. Suppose that $T$ is squarefree and $W$ a closed $\mathrm{GL}_{d}$-stable subvariety of the classical variety $\operatorname{Mod}_{d}^{T}$ of d-dimensional modules with top $T$. Let $V$ be the 
corresponding closed $\operatorname{Aut}_{\Lambda}(P)$-stable subvariety of $\mathfrak{G r a s s}_{d}^{T}$ (under the bijection of Proposition 2.5 - in particular, $W=\operatorname{Mod}_{d}^{T}$ implies $\left.V=\mathfrak{G r a s s}_{d}^{T}\right)$. Then the following statements are equivalent:

(1) There exists a fine moduli space for the isomorphism classes of modules represented by $W$, i.e., for the isomorphism classes $R(x), x \in W$.

(2) There exists a coarse moduli space for the isomorphism classes of modules represented by $W$.

(3) $V$ is the fine moduli space for the modules represented by $W$.

(4) $W$ has a geometric quotient modulo $\mathrm{GL}_{d}$.

(5) All $R(x)$ with $x \in W$ are devoid of top-stable degenerations.

(6) All $C \in V$ are fully invariant in $P$.

In case the modules represented by $W$ possess a moduli space, they all split into local direct summands. Moreover, the restriction to $V$ of the family $(\Gamma, \gamma)$ over $\mathfrak{G r a s s}_{d}^{T}$ constructed in Lemma 3.3 is the universal family in this situation.

In particular, if the d-dimensional modules with top $T$ have a moduli space classifying them up to isomorphism, then the universal family of such modules is parametrized by $\mathfrak{G r a s s}_{d}^{T}$ and restricts to the trivial bundle on each of the affine patches $\mathfrak{G} \mathfrak{r a s s}(\sigma)$.

Proof. (3) $\Longrightarrow(1) \Longrightarrow(2)$ is trivial, as is $(4) \Longrightarrow(5)$. Moreover, (6) $\Longrightarrow(4)$ is due to the fact that (6) makes $V$ its own geometric quotient modulo $\operatorname{Aut}_{\Lambda}(P)$; by Proposition 2.5, (4) follows. The equivalence of (5) and (6) can be obtained from Theorem 4.2. That (2) implies (5) can be deduced from Criterion 2.1 (just restrict the locally universal bundle over $\operatorname{Mod}_{d}^{T}$ to $W$ ).

$(6) \Longrightarrow(3)$. Adopt (6), which means that all $\operatorname{Aut}_{\Lambda}(P)$-orbits of $V$ are reduced to points. Then $V$ is its own geometric quotient modulo $\operatorname{Aut}_{\Lambda}(P)$ and, using Criterion 2.6, we infer that $V$ is a coarse moduli space for the families of modules isomorphic to $P / C$ with $C \in V$. To see that $V$ is even a fine moduli space, we restrict the family $(\Gamma, \gamma)$ parametrized by $\mathfrak{G r a s s}_{d}^{T}$, constructed in Lemma 3.3, to the subvariety $V$ and show that this latter family is universal. So let $(\Delta, \delta)$ be any family of $d$-dimensional modules recruited from the $P / C$ with $C \in V$, where $\Delta$ is a vector bundle over a variety $X$, say. Define a map $\tau: X \rightarrow V \subseteq \mathfrak{G r a s s}_{d}^{T}$ by sending any point $x$ in $X$ to the unique point $C \in \mathfrak{G r a s s}_{d}^{T}$ with the property that the fibre of $\Delta$ above $x$ is $\Lambda$-isomorphic to $P / C$. In view of the fact that $V$ is already known to be a coarse moduli space, meaning that the bijection $\alpha=\left.\phi\right|_{V} ^{-1}$ from the set of isomorphism classes of modules $P / C$ with $C \in V$ to the variety $V$ satisfies the conditions of $1.6^{\prime}$ in [16, p.24], it is now routine to check that $\tau$ is a morphism and $(\Delta, \delta)$ is equivalent to $\tau^{*}\left(\left.(\Gamma, \gamma)\right|_{V}\right)$. Obviously, $\tau$ is unique with this property.

The final statements follow from Theorem 4.2 and the previous paragraph, respectively.

Squarefreeness of $T$ cannot be discarded from the hypotheses of Theorem 4.4. To back this up, we again refer to Example 3.7. Namely, let $W \subseteq \operatorname{Mod}_{d}^{T}$ be the $\mathrm{GL}_{d}$-orbit representing the module $M=\Lambda e_{1} \oplus S_{1}$. Then $W$ is closed in $\operatorname{Mod}_{d}^{T}$, but, while $W$ has a trivial geometric quotient modulo $\mathrm{GL}_{d}$, condition (6) of the theorem is violated.

Theorem 4.4 shows, in particular, that the isomorphism classes of $d$-dimensional modules with top $T$ have a fine moduli space provided that the simple summands of $T$ do not recur as composition factors of $J P /(\operatorname{soc} J P)$. As was pointed out 
to the author by Crawley-Boevey, the following special case can also be derived from King's work [12. Namely, if $T=\Lambda e / J e$ is simple and $e J e=0$, then, for any $d \in \mathbb{N}$, the modules with top $T$ and fixed class in $K_{0}(\Lambda)$ - that is, with fixed dimension vector - possess a fine moduli space. (Indeed, if the total dimension is $d$ and $\Theta: K_{0}(\Lambda) \rightarrow \mathbb{Z}$ is the $\mathbb{Z}$-linear map which sends the class of $T$ to $-(d-1)$ and all other simples to 1 , then the modules specified are $\Theta$-stable.)

The situation where, for all $d$, the $d$-dimensional modules with fixed simple top $T$ have a fine moduli space, can be characterized in terms of the quiver and the relations of $\Lambda$, a justification being immediate from Theorem 4.4.

Corollary 4.5. Given a simple module $T=\Lambda e / J e$, the following statements are equivalent:

(a) For all d, the d-dimensional modules with top $T$ have a coarse moduli space.

(b) For all d, the variety $\mathfrak{G} \mathfrak{r a s s}_{d}^{T}$ is a fine moduli space for the d-dimensional modules with top $T$.

(c) Every left ideal $C \subseteq \Lambda$ e is of the form $C=C \Lambda \cap \Lambda$ e.

(d) For every element $\lambda \in J e$ and every oriented cycle $\omega \in$ eJe, the product $\lambda \omega$ belongs to $\Lambda \lambda$.

Clearly, these conditions are satisfied whenever $(J e)^{2}=0$, a fortiori when eJe $=$ 0.

To further illustrate the richness of the representation theory in situations where the modules with fixed top are classifiable, we mention that arbitrary irreducible projective varieties occur (up to isomorphism) as irreducible components of moduli spaces $\mathfrak{G r a s s}_{d}^{T}$ for families of modules with fixed dimension $d$ and fixed top $T$. The method of [9. Theorem $\mathrm{G}$ ] can be adapted to show that each projective variety $V$ can be realized as a fine moduli space of uniserial modules with a fixed sequence $\mathbb{S}=(S(1), \ldots, S(d))$ of consecutive composition factors. This was done by L. Hille, to whom the author had communicated her construction method prior to publication (see [8], Example). In Hille's example, $\mathfrak{G} \mathfrak{r a s s}(\mathbb{S})$ is a union of irreducible components of the encompassing variety $\mathfrak{G r a s s}_{d}^{T}$ (in general, this not the case; see [1]).

4.B. Remarks on quotients of $\mathfrak{G r a s s}(\mathbb{S})$. As one might suspect, there is a plethora of cases where the $d$-dimensional modules with top $T$ do not possess a moduli space, whereas, for each $d$-dimensional semisimple sequence with top $T$, the modules with radical layering $\mathbb{S}$ do. The simplest example illustrating this fact is Example 2.4: if $\mathbb{S}=\left(S_{1}, S_{1}, S_{2}\right)$ and $\mathbb{S}^{\prime}=\left(S_{1}, S_{1} \oplus S_{2}, 0\right)$, each of the two subvarieties $\mathfrak{G r a s s}(\mathbb{S})$ and $\mathfrak{G} \mathfrak{r a s s}\left(\mathbb{S}^{\prime}\right)$ trivially has a moduli space (a singleton in each case), whereas $\mathfrak{G r a s s}_{3}^{S_{1}}=\mathfrak{G} \mathfrak{r a s s}(\mathbb{S}) \cup \mathfrak{G r a s s}\left(\mathbb{S}^{\prime}\right)$ does not (by Theorem 4.4).

From Criterion 2.6, we know that the representations with radical layering $\mathbb{S}$ have a coarse moduli space precisely when the $\operatorname{Aut}_{\Lambda}(P)$-space $\mathfrak{G r a s s}(\mathbb{S})$ has a categorical quotient which is an orbit map. Obviously, closedness of the $\operatorname{Aut}_{\Lambda}(P)$-orbits in $\mathfrak{G} \mathfrak{r a s s}(\mathbb{S})$ (equivalently, absence of proper layer-stable degenerations among the modules with radical layering $\mathbb{S}$ ) is a necessary condition for this event. In view of Corollary 4.3, it is automatically satisfied when $T$ is simple (but not so for more general $T$; see [10]). Yet, even for a sequence $\mathbb{S}$ with simple top $T$, the modules with radical layering $\mathbb{S}$ frequently fail to possess a coarse moduli space. Indeed, another obstacle is as follows. Assume, for the moment, that $\mathfrak{G} \mathfrak{r a s s}(\mathbb{S})$ is irreducible and 
$\pi: \mathfrak{G r a s s}(\mathbb{S}) \rightarrow \mathfrak{G r a s s}(\mathbb{S}) / \operatorname{Aut}_{\Lambda}(P)$ is a categorical quotient which is an orbit map. Then $\pi$ is dominant, and hence, by [7, AG.10.1], all $\operatorname{Aut}_{\Lambda}(P)$-orbits of $\mathfrak{G} \mathfrak{r a s s}(\mathbb{S})$ have the same dimension. For an example where this condition is violated, let $l \geq 2$ and consider the algebra

$$
\Lambda=K Q /\langle\text { all paths of length } l+1\rangle
$$

where $Q$ is the quiver

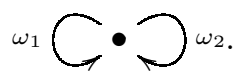

If $S$ is the unique simple left $\Lambda$-module and $\mathbb{S}$ the $(l+1)$-dimensional semisimple sequence $(S, S, \ldots, S)$, then $\mathfrak{G r a s s}(\mathbb{S})=\left(\mathbb{P}^{1}\right)^{l} \times \mathbb{A}^{l(l-1) / 2}$ is irreducible, but all numbers between 0 and $l-1$ arise as dimensions of $\operatorname{Aut}_{\Lambda}(P)$-orbits of $\mathfrak{G r a s s}(\mathbb{S})$; for details, see [5], Example, p. 27.

On the other hand, we believe such skips in the orbit dimension to be the only impediment (beyond nonclosedness of the orbits for nonsimple $T$ ) in the way of a moduli space. In fact, a variety of examples led us to the following sharper conjecture.

Conjecture 4.6. The following conditions are equivalent for a semisimple sequence $\mathbb{S}$ with squarefree top $T$ :

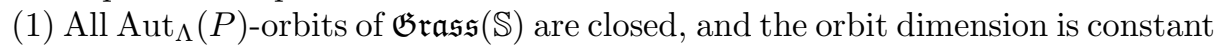
on each irreducible component of $\mathfrak{G} \mathfrak{r a s s}(\mathbb{S})$.

(2) There exists a coarse moduli space for the representations with radical layering $\mathbb{S}$.

(3) There exists a fine moduli space for the representations with radical layering $\mathbb{S}$.

$(4) \mathfrak{G r a s s}(\mathbb{S})$ has a geometric quotient modulo $\operatorname{Aut}_{\Lambda}(P)$.

If $T$ is simple, the first requirement under (1) is automatic, and the second is equivalent to the dimension of the endomorphism $\operatorname{rings} \operatorname{End}_{\Lambda}(P / C)$ being constant, where $C$ traces an irreducible component of $\mathfrak{G} \mathfrak{r a s s}(\mathbb{S})$.

\section{Algebras of finite local Representation type}

Our main objective in this section is to characterize the algebras which, up to isomorphism, permit only finitely many modules with a fixed simple top $T$. We start

by considering an invariant that measures the size of the category $\operatorname{Add}_{\Lambda}^{T}$ consisting of the direct sums of left $\Lambda$-modules with top $T$. This invariant fails to be left-right symmetric in general.

Definition 5.1. Given a simple left $\Lambda$-module $T$ with projective cover $P$, the local dimension of $\Lambda$ - $\bmod$ at $T$, denoted

$$
\operatorname{loc} \operatorname{dim}^{T}(\Lambda-\bmod ),
$$

is the maximum of the following differences:

$$
\operatorname{dim} \mathcal{C}-\text { generic fibre dimension of } \phi \text { on } \mathcal{C},
$$

where $\mathcal{C}$ runs through the irreducible components of the varieties $\mathfrak{G r a s s}_{d}^{T}$ for $1 \leq$ $d \leq \operatorname{dim}_{K} P$. (Recall that $\phi$ stands for the pertinent representation map.) 
The local dimension of $\Lambda$ at $T$ can be obtained from the $\operatorname{Aut}_{\Lambda}(P)$-stable affine covers of the varieties $\mathfrak{G r a s s}_{d}^{T}$ for $d \geq 1$. Indeed, $\operatorname{loc} \operatorname{dim}^{T}(\Lambda$ - mod) equals the maximum of the differences ' $\operatorname{dim} \mathcal{D}$ - generic $\operatorname{Aut}_{\Lambda}(P)$-orbit dimension on $\mathcal{D}$ ', where $\mathcal{D}$ traces the irreducible components of the $\mathfrak{G} \mathfrak{r a s s}(\sigma)$ and $\sigma$ ranges through all skeletons with top $T$. For a computation of the generic fibre dimensions on the irreducible components $\mathcal{D}$, a fairly small system of linear equations suffices. Yet, in checking whether $\operatorname{loc} \operatorname{dim}^{T}(\Lambda-\bmod )=0$ for an algebra $\Lambda$ presented in terms of a quiver and relations, it is usually more convenient to use one of the last two of the equivalent conditions presented in Theorem 5.2 below and combine the procedures of Section 3.B with Gröbner methods. In case $\Lambda$ - mod has vanishing local dimension at $T$, all local modules with top $T$ can be computed from quiver and relations of $\Lambda$. Due to the theorem, they are classified by their radical layerings. Our concluding example demonstrates that, by contrast, dimension vectors do not separate nonisomorphic modules in general.

Theorem 5.2. For any simple left $\Lambda$-module $T$ with projective cover $P$, the following statements are equivalent:

(1) There are only finitely many left $\Lambda$-modules with top $T$, up to isomorphism.

(2) $\operatorname{loc}_{\operatorname{dim}}^{T}(\Lambda-\bmod )=0$.

(3) If $M$ and $N$ are any $\Lambda$-modules with top $T$, then $\mathbb{S}(M)=\mathbb{S}(N)$ implies $M \cong N$.

(4) For every semisimple sequence $\mathbb{S}$ with top $T$, the variety $\mathfrak{G} \mathfrak{r a s s}(\mathbb{S})$ is either empty or irreducible of dimension

$$
\mu_{T}(M)-\operatorname{dim}_{K} \operatorname{End}_{\Lambda}(M)
$$

for some (equivalently, for all) $M$ with radical layering $\mathbb{S}$.

(5) For every skeleton $\sigma$ with top $T$, the variety $\mathfrak{G} \mathfrak{r a s s}(\sigma)$ is either empty or consists of a single $\operatorname{Aut}_{\Lambda}(P)$-orbit.

If these equivalent conditions are satisfied, the varieties $\mathfrak{G} \mathfrak{r a s s}(\mathbb{S})$, where $\mathbb{S}$ ranges through the semisimple sequences with top $T$, are either empty or isomorphic to full affine spaces. If, in addition, $Q$ does not contain oriented cycles, then each $\mathfrak{G} \mathfrak{r a s s}(\mathbb{S})$ is either empty or a point.

Proof. (1) $\Longrightarrow(2)$. Adopt (1) and assume $\mathbb{S}$ to be a semisimple sequence with top $T$ such that $\mathfrak{G} \mathfrak{r a s s}(\mathbb{S}) \neq \varnothing$. If $\mathcal{C}$ is an irreducible component of $\mathfrak{G} \mathfrak{r a s s}(\mathbb{S})$, then $\mathcal{C}$ consists of only finitely many fibres of $\phi$ by hypothesis. All of them are closed in $\mathfrak{G} \mathfrak{r a s s}(\mathbb{S})$ by Corollary 4.3, and a fortiori closed in $\mathcal{C}$. Therefore $\mathcal{C}$ consists of a single fibre. In particular, $\operatorname{dim} \mathcal{C}$ equals the generic fibre dimension of $\phi$ on $\mathcal{C}$. Since, for each $d$, the irreducible components of $\mathfrak{G r a s s}_{d}^{T}$ are among the closures, in $\mathfrak{G r a s s}_{d}^{T}$, of the irreducible components of the $\mathfrak{G} \mathfrak{r a s s}(\mathbb{S})$ 's covering $\mathfrak{G r a s s}_{d}^{T}$, we conclude that $\operatorname{loc} \operatorname{dim}^{T}(\Lambda-\bmod )=0$.

$(2) \Longrightarrow(1)$. Given (2), it suffices to prove that, for any semisimple sequence $\mathbb{S}$ with top $T$, there are only finitely many modules $M$ with $\mathbb{S}(M)=\mathbb{S}$ up to isomorphism; indeed, there are just finitely many such sequences $\mathbb{S}$, since their total dimensions are all bounded by $\operatorname{dim}_{K} P$. Assume $\mathfrak{G} \mathfrak{r a s s}(\mathbb{S}) \neq \varnothing$ and let $\mathcal{C}$ be an irreducible component of $\mathfrak{G r a s s}(\mathbb{S})$. If $C \in \mathcal{C}$ has an $\operatorname{Aut}_{\Lambda}(P)$-orbit of maximal dimension, then $\operatorname{dim}_{\operatorname{Aut}_{\Lambda}}(P) \cdot C=\operatorname{dim} \mathcal{C}$ by hypothesis, and since $\operatorname{Aut}_{\Lambda}(P) \cdot C$ is irreducible and closed in $\mathfrak{G} \mathfrak{r a s s}(\mathbb{S})$ by Proposition 4.1, we infer that $\mathcal{C}=\operatorname{Aut}_{\Lambda}(P) . C$, i.e., $\phi(\mathcal{C})$ is a singleton. 
$(1) \Longrightarrow(3)$. We assume $(1)$, let $\mathbb{S}=\left(\mathbb{S}_{0}, \ldots, \mathbb{S}_{L}\right)$ be any semisimple sequence with top $T$ such that $\mathfrak{G r a s s}(\mathbb{S})$ is nonempty, and $C \in \mathfrak{G r a s s}(\mathbb{S})$. We show by induction on the dimension $d$ of $\mathbb{S}$ that $M=P / C$ is the only local module with semisimple sequence $\mathbb{S}$, up to isomorphism. Let $k$ be the largest integer with $\mathbb{S}_{k} \neq 0$. Without loss of generality, $d \geq 2$, which means that $k \geq 2$, because $\operatorname{dim}_{K} \mathbb{S}_{0}=$ $\operatorname{dim}_{K} T=1$. Then $M^{\prime}=M / J^{k} M$ is local with semisimple sequence $\mathbb{S}\left(M^{\prime}\right)=$ $\left(\mathbb{S}_{0}, \ldots, \mathbb{S}_{k-1}, 0, \ldots, 0\right)$, and by the induction hypothesis, $M^{\prime}$ is unique with this property. This focuses our attention on the following subset of $\operatorname{Mod}_{d}^{\Lambda}$, namely on

$$
\text { Ext }=\left\{x \in \operatorname{Mod}_{d}^{\Lambda} \mid \exists \text { an exact sequence } 0 \rightarrow \mathbb{S}_{k} \rightarrow R(x) \rightarrow M^{\prime} \rightarrow 0\right\} .
$$

By [2, 6.3], Ext is an irreducible subset of $\operatorname{Mod}_{d}^{\Lambda}$. Therefore $\operatorname{Ext} \cap \mathbf{M o d}_{d}^{T}$ is irreducible as well; indeed, due to the upper semicontinuity of the maps $\operatorname{dim}_{K} \operatorname{Hom}_{\Lambda}\left(-, S_{r}\right)$, this intersection is open in Ext. Moreover, our construction guarantees that

$$
\operatorname{Mod}(\mathbb{S})=\operatorname{Ext} \cap \operatorname{Mod}(\mathbb{S})=\left(\operatorname{Ext} \cap \operatorname{Mod}_{d}^{T}\right) \backslash\left(\bigcup_{\underline{\operatorname{dim}} \mathbb{S}^{\prime}>\underline{\operatorname{dim}} \mathbb{S}} \operatorname{Mod}\left(\mathbb{S}^{\prime}\right)\right)
$$

Since the union $\bigcup_{\underline{\operatorname{dim}}} \mathbb{S}^{\prime}>\underline{\operatorname{dim}} \mathbb{S} \operatorname{Mod}\left(\mathbb{S}^{\prime}\right)$ is closed in $\operatorname{Mod}_{d}^{T}$ by Lemma 2.8(i) and Proposition 2.5, we infer that $\operatorname{Mod}(\mathbb{S})$ is again irreducible. Hence so is $\mathfrak{G} \mathfrak{r a s s}(\mathbb{S})$ by Proposition 2.5. In light of our hypothesis, $\mathfrak{G r a s s}(\mathbb{S})$ consists of finitely many orbits, one of which is $\operatorname{Aut}_{\Lambda}(P) . C$, and the fact that all of these orbits are closed in $\mathfrak{G r a s s}(\mathbb{S})$ by part $(3)$ of Proposition 4.1 thus yields $\mathfrak{G r a s s}(\mathbb{S})=\operatorname{Aut}_{\Lambda}(P) . C$. This finishes the induction and establishes (3).

$(3) \Longleftrightarrow(4)$ is immediate from Proposition 4.1(3), as is the fact that (3) implies the supplementary statement. The implications $(3) \Longrightarrow(5) \Longrightarrow(2)$ are obvious.

As a special case we recover the following result of Bongartz in [3]:

Corollary 5.3. Let $\mathbb{S}=\left(\mathbb{S}_{0}, \ldots, \mathbb{S}_{L}\right)$ be a semisimple sequence with the property that each nonzero $\mathbb{S}_{i}$ is simple. If $\Lambda$ has only finitely many uniserial modules, up to isomorphism, there is at most one uniserial left $\Lambda$-module with sequence $\mathbb{S}$ of consecutive composition factors.

The following extension of Example 2.4 shows that, even in case $\operatorname{loc} \operatorname{dim}^{T}(\Lambda$ - mod) $=0$, the varieties $\mathfrak{G}_{\mathfrak{r a s s}} \mathfrak{s}_{d}^{T}$ may have arbitrarily large dimension.

Example 5.4. For any $m \geq 1$, we present a finite-dimensional algebra $\Lambda$, together with a simple left $\Lambda$-module $T$, such that $\operatorname{loc}_{\operatorname{dim}}^{T}(\Lambda$ - mod $)=0$ while $\mathfrak{G r a s s}_{m+2}^{T}$ contains a copy of $\mathbb{P}^{m}$. Let $\Lambda=Q / I$, where $Q$ is the quiver

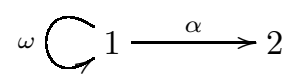

and $I$ the ideal generated by $\omega^{m+1}$. Moreover, let $T=S_{1}$. Using Theorem 5.2, one readily verifies that $\operatorname{loc} \operatorname{dim}^{T}(\Lambda$ - mod $)=0$. Via the methods of [10], one moreover checks that $\mathfrak{G} \mathfrak{r a s s}_{m+2}^{T}$ contains precisely $m+1$ distinct orbits, all representing modules with dimension vector $(m+1,1)$; these are isomorphic to $\mathbb{A}^{0}, \mathbb{A}^{1}, \ldots, \mathbb{A}^{m}$ and correspond to the submodules $C_{j}=\sum_{0 \leq i \leq m, i \neq j} \Lambda \alpha \omega^{i}$ of $\Lambda e_{1}$, respectively. From Lemma 2.8 and Theorem 4.2 one thus derives by induction that the orbit closures are stacked into one another as follows:

$$
\overline{\operatorname{Aut}_{\Lambda}(P) \cdot C_{j}}=\bigcup_{i \leq j} \operatorname{Aut}_{\Lambda}(P) \cdot C_{i} .
$$


Here is a sketch: Given that $\operatorname{Aut}_{\Lambda}(P) \cdot C_{1} \cong \mathbb{A}^{1}$, this orbit fails to be closed, and hence its closure is $\operatorname{Aut}_{\Lambda}(P) \cdot C_{1} \cup \operatorname{Aut}_{\Lambda}(P) \cdot C_{0}$. Similarly, the closure of $\operatorname{Aut}_{\Lambda}(P) \cdot C_{2}$ is seen to contain $\operatorname{Aut}_{\Lambda}(P) . C_{1}$, and so on. In particular, we conclude that the closure of $\operatorname{Aut}_{\Lambda}(P) \cdot C_{m}$ is isomorphic to $\mathbb{P}^{m}$.

\section{Proof of Theorem 3.14}

We start by showing that $\chi_{\sigma}$ is well defined and surjective and then check that $\psi_{\sigma}$ is a bijective set map. That $\psi_{\sigma}$ is actually an isomorphism of varieties can be verified by an argument modeled on that of Bongartz and the author in 5 , Theorem A]; we leave it to the reader to make the obvious modifications. That the triangle of maps commutes is obvious.

It will be convenient to write $\sigma^{(r)}$ for the set of paths in $\sigma$ which start in the vertex $e_{r}$ and to let $\sigma_{l}$, resp. $\sigma_{l}^{(r)}$, be the subset of $\sigma$, resp. of $\sigma^{(r)}$, consisting of the paths of length $l$; accordingly, $\sigma_{l}=\bigcup_{1 \leq r \leq t} \sigma_{l}^{(r)}$ and $\sigma=\bigcup_{0 \leq l \leq L} \sigma_{l}$.

For well-definedness of $\chi_{\sigma}$, we need to show that, for any point $c \in \operatorname{Aff}(\sigma)$, the factor module $M(c)=P / U(c)$ has skeleton $\sigma$. Set $x_{r}=e_{r}+U(c) \in M(c)$ for $1 \leq r \leq t$. Clearly, $M(c)$ is generated by the elements $p x_{r}$ for $r \leq t$ and $p \in \sigma^{(r)}$. Indeed, with the argument backing Proposition 3.12, one checks that, given any path $q$ and $r \leq t$, the element $q x_{r}$ of $M(c)$ is a $K$-linear combination of terms $p x_{s}$ with $p \in \sigma^{(s)}, s \leq t$. So it suffices to verify that, for each $l \in\{0, \ldots, L\}$, the subset $\bigcup_{1 \leq r \leq t}\left\{p x_{r} \mid p \in \sigma_{l}^{(r)}\right\}$ of $M(c)$ is linearly independent modulo $J^{l+1} M(c)$. On the assumption that this fails, let $l_{0}$ be minimal with respect to failure for some factor algebra of $\Lambda$ and some point $c$ in a suitable $\operatorname{Aff}(\sigma)$. It is harmless to assume that $l_{0}=L$. For otherwise we can enlarge the ideal $I \subseteq K Q$ so as to include all paths of length $l_{0}+1$, replace $\sigma$ by $\left(\bigcup_{l \leq l_{0}} \sigma_{l}\right)$, and let $d$ be the cardinality of this union; in this modified setup $l_{0}$ is still minimal with respect to failure of our independence condition. So we only need to refute the assumption that the set $\sigma_{L}^{(1)} x_{1} \cup \cdots \cup \sigma_{L}^{(t)} x_{t}$ is linearly dependent in $M(c)$.

To obtain a convenient framework for a comparison of coefficients, consider the projective left ideal $\widehat{P}:=K Q e_{1} \oplus \cdots \oplus K Q e_{t}$ of $K Q$ and note that $\widehat{P} / I \widehat{P} \cong$ $P$. Furthermore, we let $V(c)$ be the submodule of $\widehat{P}$ generated by the differences $\alpha p-\sum_{q \in \sigma(\alpha, p)} c_{\alpha p, q} q$, where $(\alpha, p)$ ranges through the $\sigma$-critical pairs. Viewed as a $K Q$-module, $M(c)$ is then isomorphic to the quotient $\widehat{P} /(V(c)+I \widehat{P})$.

Next, we note that, for $1 \leq r \leq t$, the set of paths in $K Q e_{r}$ is the disjoint union of the following two sets: the set $\sigma^{(r)}$, and that of all paths of the form $u \alpha p$, where $(\alpha, p)$ is a $\sigma$-critical pair with $p \in \sigma^{(r)}$ and $u$ a path of length $\geq 0$. This permits us to define a $K$-linear transformation

$$
F_{c}: \widehat{P} \longrightarrow \widehat{P}
$$

as follows. Suppose that $p$ is any path starting at one of the vertices $e_{r}$ for $r \leq t$. We set $F_{c}(p)=p$ if $p \in \sigma^{(r)}$; moreover, we define $F_{c}(u \alpha p)=\sum_{q \in \sigma(\alpha, p)} c_{\alpha p, q} u q$ in the second case. Our choice of $c$ in $\operatorname{Aff}(\sigma)$ guarantees that $F_{c}^{L}(I \widehat{P})=0$. Indeed, if $v$ is any path, of length $l$ say, Proposition 3.12 yields a congruence

$$
v \widehat{=} \sum_{q \in \sigma} \tau_{q}^{v}(X) q
$$


in the polynomial ring $\mathcal{A}$ over $K Q$, where the $\tau_{q}^{v}(X)$ are suitable polynomials in $K\left[X_{\alpha p, q}\right]$. Along the induction backing Proposition 3.12, we then find $F_{c}^{l}(v)=$ $\sum_{q \in \sigma} \tau_{q}^{v}(c) q$. Hence, if $\rho \in I \widehat{P}=I e_{1}+\cdots+I e_{t}$, then $F_{c}^{L}(\rho)=\sum_{q \in \sigma} \tau_{q}^{\rho}(c) q=0$ because the $\tau_{q}^{\rho}(X)$ annihilate all points in $\operatorname{Aff}(\sigma)$ by construction. It follows that $F_{c}^{L}(I \widehat{P})=0$. Next we observe that, for each element $z \in \widehat{P}$, the difference $z-F_{c}(z)$ belongs to $V(c)$, which makes $V(c)$ invariant under $F_{c}$.

By assumption, there is a finite list $p_{1}, \ldots, p_{s}$ of distinct paths in $\sigma$, say $p_{i} \in$ $\sigma^{\left(r_{i}\right)}$, together with nonzero scalars $k_{1}, \ldots, k_{s}$, such that the linear combination $\sum_{1 \leq i \leq s} k_{i} p_{i} x_{r_{i}}$ vanishes in $M(c)$. On moving to the above presentation of $M(c)$ as a left $K Q$-module, we infer that the element $\sum_{1 \leq i \leq s} k_{i} p_{i}$ of $\widehat{P}$ is a sum $z+a$ with $z \in V(c)$ and $a \in I \widehat{P}$. Now $F_{c}^{L}$ annihilates $a$ and maps $z$ back to $V(c)$, while leaving the sum $\sum_{1 \leq i \leq s} k_{i} p_{i}$ fixed, thus yielding $\sum_{1 \leq i \leq s} k_{i} p_{i} \in V(c)$. Since any path including a nonroute on $\sigma$ as a right subpath is again a nonroute, this yields an equality

$$
\sum_{1 \leq i \leq s} k_{i} p_{i}=\sum_{(\alpha, p)} \sum_{\sigma \text {-critical }} b_{k \geq 1} b_{\alpha p, k} u_{\alpha p, k}\left(\alpha p-\sum_{q \in \sigma(\alpha, p)} c_{\alpha p, q} q\right)
$$

for suitable scalars $b_{\alpha p, k}$ and paths $u_{\alpha p, k}$ of lengths $\geq 0$, starting at the endpoint of $\alpha$, respectively; clearly, we may assume that $u_{\alpha p, j} \neq u_{\alpha p, k}$ for $j \neq k$. This being an equality in $\widehat{P}$, we are now in a position to compare coefficients.

In doing this, the concept of a route on $\sigma$, introduced in Remark 3.15, will come in handy. Since none of the paths $u_{\alpha p, k} \alpha p$ on the right-hand side of ( $\dagger$ ) belongs to $\sigma$, each $p_{i}$ on the left-hand side must equal one of the paths $u_{\alpha p, k} q$. Moreover, one observes that, whenever we have an equality $p_{i}=u_{\alpha p, k} q$ for some $q \in \sigma(\alpha, p)$, the path $u_{\alpha p, k} \alpha p$ is a route on $\sigma$. We can therefore find a $\sigma$-critical pair $\left(\alpha_{0}, p_{0}\right)$ such that $p_{0} \in \sigma$ has minimal length with respect to the following property: there exists an index, say $k=1$, with

- $b_{\alpha_{0} p_{0}, 1} \neq 0$ and such that

- $u_{\alpha_{0} p_{0}, 1} \alpha_{0} p_{0}$ is a route on $\sigma$.

Set $w=u_{\alpha_{0} p_{0}, 1} \alpha_{0} p_{0}$ and note that $w \notin \sigma$. The left-hand side of ( $\dagger$ ) being a $K$ linear combination of paths in $\sigma$, the path $w$ must cancel out of the right-hand side. Observe that $w$ does not equal any path of the form $u_{\alpha p, k} \alpha p$ with $(\alpha, p) \neq\left(\alpha_{0}, p_{0}\right)$, for $p_{0}$ is the longest right subpath of $w$ which belongs to $\sigma$; nor does $w$ coincide with one of the paths $u_{\alpha_{0} p_{0}, k} \alpha_{0} p_{0}$ for $k \neq 1$. Consequently, $w$ must be one of the $u_{\alpha p, k} q$ for some $\sigma$-critical pair $(\alpha, p)$, some choice of $q \in \sigma(\alpha, p)$, and some $k$ with $b_{\alpha p, k} \neq 0$. In particular, this makes $u_{\alpha p, k} q$ a route on $\sigma$, which entails that $u_{\alpha p, k} \alpha p$ is also a route on $\sigma$. We infer that length $(p) \geq \operatorname{length}\left(p_{0}\right)$ by the minimality of length $\left(p_{0}\right)$, and further deduce that length $(q)>\operatorname{length}\left(p_{0}\right)$ because $q$ belongs to $\sigma(\alpha, p)$. On the other hand, the equality

$$
w=u_{\alpha_{0} p_{0}, 1} \alpha_{0} p_{0}=u_{\alpha p, k} q
$$

implies that length $\left(p_{0}\right) \geq$ length $(q)$, once again due to the maximal length of $p_{0}$ as a right subpath of $w$ that belongs to $\sigma$. This contradiction shows our original assumption $\sum_{i \leq s} k_{i} p_{i} x_{r_{i}}=0$ to be absurd. Hence $\sigma$ is a skeleton of $M(c)$ as claimed, which proves the well-definedness of $\chi_{\sigma}$.

To see that $\chi_{\sigma}$ is a surjection, let $M$ be any $\Lambda$-module with skeleton $\sigma$; in particular, this ensures that $M$ is $d$-dimensional with top $T$. Choose a sequence 
$y_{1}, \ldots, y_{t}$ of top elements of $M$ with $y_{r}=e_{r} y_{r}$. For each $l$, the products $p y_{r}$ for $p \in \sigma_{l}^{(r)}$ and $1 \leq r \leq t$ then form a basis for $J^{l} M / J^{l+1} M$. Moreover, for any $\sigma$-critical pair $(\alpha, p)$, with $p \in \sigma^{(r)}$ say, let $c_{\alpha p, q}$ be the unique scalars such that $\alpha p y_{r}=\sum_{s \leq t} \sum_{q \in \sigma(\alpha, p) \cap \sigma^{(s)}} c_{\alpha p, q} q y_{s}$. It clearly suffices to show that the corresponding point $c=\left(c_{\alpha p, q}\right) \in \mathbb{A}^{N}$ belongs to the variety $\operatorname{Aff}(\sigma)$, for it is then clear that $M \cong P / U(c)$; just send $y_{r}$ to $e_{r}+U(c)$. So let us check that $c \in \operatorname{Aff}(\sigma)$. For that purpose, suppose $v \in I e_{r}$ for some $r$ between 1 and $t$. Viewing $v$ as an element of $\mathcal{A}$, consider the expansion $v \widehat{=} \sum_{q \in \sigma} \tau_{q}^{v}(X) q$ with $\tau_{q}^{v}(X) \in K[X]$ supplied by Proposition 3.12. In tandem with the inductive procedure justifying the lemma, one obtains the following equalities in $M$ :

$$
v y_{r}=\sum_{s \leq t} \sum_{q \in \sigma^{(s)}} \tau_{q}^{v}(c) q y_{s}
$$

here we refer to the induced $K Q$-structure of $M$. Consequently, the linear independence of the elements $q y_{s}$, for $q \in \sigma^{(s)}$ and $s \leq t$, yields vanishing of all the values $\tau_{q}^{v}(c)$. This means that $c \in \operatorname{Aff}(\sigma)$ as asserted and shows that $\chi_{\sigma}$ is indeed a surjection.

That the map $\psi_{\sigma}$ is injective follows from the first part of the proof; indeed, it suffices to observe that, for any $c \in \operatorname{Aff}(\sigma)$, the paths in $\sigma$ are linearly independent modulo $U(c)$. To verify surjectivity, let $C \in \mathfrak{G} \mathfrak{r a s s}(\sigma)$. Then $P / C$ has skeleton $\sigma$ and top elements $e_{r}+C$. Therefore, as explained in the surjectivity argument for $\psi_{\sigma}$, there exists a point $c \in \operatorname{Aff}(\sigma)$ with the property that, for any $\sigma$-critical pair $(\alpha, p)$ with $p \in \sigma^{(r)}$,

$$
\alpha p\left(e_{r}+C\right)=\sum_{s \leq t} \sum_{q \in \sigma(\alpha, p) \cap \sigma^{(s)}} c_{\alpha p, q} q\left(e_{s}+C\right) .
$$

In other words, $U(c) \subseteq C$. But we already know that $U(c)$ has codimension $d$ in $P$ as does $C$, and hence $\psi_{\sigma}(c)=U(c)=C$. This completes the proof of Theorem 3.14 .

\section{Moduli spaCes FOR REPRESEntations With ARBitrary top $T$ : A PREVIEW}

Suppose that $T=\bigoplus_{1 \leq i \leq n} S_{i}^{t_{i}}$ is an arbitrary semisimple module, with projective cover $P$. Recall from Observation 2.3 that

$$
\operatorname{Aut}_{\Lambda}(P) \cong \mathcal{U} \rtimes \operatorname{Aut}_{\Lambda}(T) \cong \mathcal{U} \rtimes \prod_{1 \leq i \leq t} \operatorname{GL}_{t_{i}}(K)
$$

where $\mathcal{U}$ denotes the unipotent radical of $\operatorname{Aut}_{\Lambda}(P)$. Moreover, let $\mathcal{T}$ be the direct product of the tori of diagonal matrices in the $\mathrm{GL}_{t_{i}}(K)$. To realize $\mathcal{T}$ as a subgroup of $\operatorname{Aut}_{\Lambda}(P)$, write $P=\bigoplus_{1<i<n} \bigoplus_{1<j<t_{i}} \Lambda x_{i j}$ for suitable elements $x_{i j}=e_{i} x_{i j}$. Then $\mathcal{T}$ can be identified with the automorphisms of $P$ sending $x_{i j}$ to $a_{i j} x_{i j}$, where $\left(a_{i j}\right)_{i \leq n, j \leq t_{i}} \in\left(K^{*}\right)^{\operatorname{dim} T}$. We observe that the equality $\operatorname{Aut}_{\Lambda}(P)=\mathcal{U} \rtimes \mathcal{T}$ holds precisely when $T$ is squarefree; it is this fact which singles out the squarefree case in the context of the moduli problem. We only give a rough picture of the general case, addressing solvability of the moduli problem for all $d$, to illustrate the strong pressure exerted by this demand on those indecomposable projective $\Lambda$-modules whose radical factors occur with multiplicity $>1$ in $T$. 
Preview 7.1. The following conditions are equivalent for the semisimple module $T$ containing the $S_{i}$ with multiplicity $t_{i}$ :

(1) For each d, all $\operatorname{Aut}_{\Lambda}(P)$-orbits of $\mathfrak{G r a s s}_{d}^{T}$ are closed.

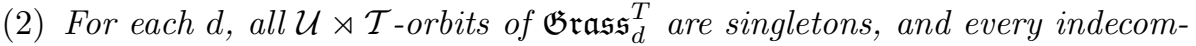
posable projective module $\Lambda e_{i}$ with $t_{i}>1$ is uniserial.

(3) For each $d$ and each point $C \in \mathfrak{G r a s s}_{d}^{T}$, the module $P / C$ is a direct sum of local modules, $\operatorname{dim} \operatorname{Hom}_{\Lambda}(P, J P / C)=\operatorname{dim}_{H_{0}}(P / C, J P / C)$, and all $\Lambda e_{i}$ with $t_{i}>1$ are uniserial.

This restricts 'global' classifiability of the representations with top $T$ to a rather narrow selection of algebras $\Lambda$. On the other hand, if one splits up the class of top- $T$ modules in terms of an invariant somewhat reminiscent of the 'words' underlying classification of the representations of biserial algebras, one obtains a fine moduli space for each of the resulting subclasses under much more general circumstances.

By a slight abuse of language, we use the term distinguished basis of a module $P / C$ for any family $\sigma=\left(\sigma_{i j}\right)_{i \leq n, j \leq t_{i}}$ of sets of paths in $K Q$ with the property that each $\sigma_{i j}$ consists of paths starting in the vertex $e_{i}$ and is closed under right subpaths (in particular, this entails $e_{i} \in \sigma_{i j}$ ) and such that, moreover, the residue classes $p x_{i j}+C$ for $p \in \sigma_{i j}$ (with $i, j$ tracing all eligible choices) form a $K$-basis of $P / C$. Clearly, each module $P / C$ with $C \subseteq J P$ has at least one such distinguished basis, and there are only finitely many families $\sigma$ that qualify as distinguished bases. Finally, when $\sum_{i, j}\left|\sigma_{i j}\right|=d$, let $\mathcal{C}(\sigma)$ be the set of all points $C$ in $\mathfrak{G r a s s}_{d}^{T}$ such that $P / C$ has a distinguished basis $\sigma$ (we refer to $\sigma$ as $d$-dimensional in that case). Note that, for each choice of $\sigma$, the set $\mathcal{C}(\sigma)$ is an open subvariety of $\mathfrak{G r a s s}_{d}^{T}$, being the intersection of a standard open affine subset of the classical Grassmannian with $\mathfrak{G}_{\mathfrak{r a s s}}{ }_{d}^{T}$. (However, the $\mathcal{C}(\sigma)$ fail to be stable under the $\mathcal{U} \rtimes \mathcal{T}$-action and, a fortiori, under the $\operatorname{Aut}_{\Lambda}(P)$-action in general.)

Preview 7.2. The following statements are equivalent for given $T$ and $d$ :

(1) For each $d$-dimensional distinguished basis $\sigma$, the family of top- $T$ modules $P / C$ with $C \in \mathcal{C}(\sigma)$ has a fine moduli space.

(2) All $\mathcal{U} \rtimes \mathcal{T}$-orbits of $\mathfrak{G r a s s}_{d}^{T}$ are singletons.

(3) For each $C \in \mathfrak{G} \mathfrak{r a s s}_{d}^{T}$, the quotient $P / C$ is a direct sum of local modules and $\operatorname{dim} \operatorname{Hom}_{\Lambda}(P, J P / C)=\operatorname{dim} \operatorname{Hom}_{\Lambda}(P / C, J P / C)$.

If the equivalent conditions of 7.2 are satisfied, each of the varieties $\mathcal{C}(\sigma)$ is the fine moduli space for the corresponding class $(P / C)_{C \in \mathcal{C}(\sigma)}$. Moreover, $\mathcal{C}(\sigma)$ is a direct product of subvarieties of Grassmannians of modules with simple tops, which brings the problem back, full circle, to the simplest squarefree case.

\section{REFERENCES}

1. E. Babson, B. Huisgen-Zimmermann, and R. Thomas, Generic representation theory of quivers with relations, in preparation.

2. K. Bongartz, On degenerations and extensions of finite dimensional modules, Advances in Math. 121 (1996), 245-287. MR1402728 (98e:16012)

3. MR.1435371 (98d:16014)

4. Some geometric aspects of representation theory, in Algebras and Modules I (I. Reiten, S.O. Smalø, and Ø. Solberg, eds.), Canad. Math. Soc. Conf. Proc. Series Vol. 23, 1998, pp. 1-27. MR.1648601 (99j:16005) 
5. K. Bongartz and B. Huisgen-Zimmermann, The geometry of uniserial representations of algebras II. Alternate viewpoints and uniqueness, J. Pure Appl. Algebra 157 (2001), 23-32. MR:1809214 (2002b:16016)

6. - Varieties of uniserial representations IV. Kinship to geometric quotients, Trans. Amer. Math. Soc. 353 (2001), 2091-2113. MR1813609 (2002h:16018)

7. A. Borel, Linear Algebraic Groups, Second enlarged ed., Springer-Verlag, New York, 1991. MR.1102012 (92d:20001)

8. L. Hille, Tilting line bundles and moduli of thin sincere representations of quivers, An. St. Univ. Ovidius Constantza 4 (1996), 76-82. MR1428456 (97h:16017)

9. B. Huisgen-Zimmermann, The geometry of uniserial representations of finite dimensional algebras I, J. Pure Appl. Algebra 127 (1998), 39-72. MR1609508 (99b:16020)

10. —, Top-stable degenerations of finite dimensional representations $I$, posted at www.math.ucsb.edu/ birge/papers.html.

11. J. E. Humphreys, Linear Algebraic Groups, Graduate Texts in Mathematics, Vol. 21, Springer-Verlag, New York, 1981. MR0396773 (53:633)

12. A. D. King, Moduli of representations of finite dimensional algebras, Quart. J. Math. Oxford 45 (1994), 515-530. MR.1315461 (96a:16009)

13. H. Kraft, Geometrische Methoden in der Invariantentheorie, Second ed., Vieweg, Braunschweig, 1985. MR0768181 (86j:14006)

14. L. Le Bruyn and A. Schofield, Rational invariants of quivers and the ring of matrix invariants, Perspectives in Ring Theory, Nato Advanced Research Workshop C-233, Kluwer Acad. Publ., Dordrecht, 1988, pp. 21-29. MR.1048393 (91f:14047)

15. D. Mumford, Geometric Invariant Theory, Springer-Verlag, Berlin, 1965 . MR0214602 (35:5451)

16. P. E. Newstead, Introduction to moduli spaces and orbit problems, Lecture Notes, Tata Institute of Fundamental Research, Springer-Verlag, Berlin-New York, 1978. MR0546290 (81k:14002)

17. Ch. Riedtmann, Degenerations for representations of quivers with relations, Ann. Sci. École Normale Sup. (4) 19 (1986), 275-301. MR0868301 (88b:16051)

18. M. Rosenlicht, On quotient varieties and the affine embedding of certain homogeneous spaces, Trans. Amer. Math. Soc. 101 (1961), 211-223. MR0130878 (24:A732)

19. _ Questions of rationality for solvable algebraic groups over nonperfect fields, Ann. Mat. Pura Appl. IV 61 (1963), 97-120. MR0158891 (28:2113)

20. A. Schofield, Birational classification of moduli spaces of representations of quivers, Indag. Math. 12 (2001), 407-432. MR1914089 (2003k:16028)

21. G. Zwara, Degenerations for modules over representation-finite algebras, Proc. Amer. Math. Soc. 127 (1999), 1313-1322. MR.1476404 (99h:16034)

Department of Mathematics, University of California, Santa Barbara, California 93106

E-mail address: birge@math.ucsb.edu 